\title{
Dopamine D1 Receptor Activation Drives Plasticity in the Songbird Auditory Pallium
}

\author{
(1) Matheus Macedo-Lima, ${ }^{1,2,3}$ Hannah M. Boyd, ${ }^{2}$ and Luke Remage-Healey ${ }^{1,2}$ \\ ${ }^{1}$ Neuroscience and Behavior Program, and ${ }^{2}$ Center for Neuroendocrine Studies, University of Massachusetts Amherst, Amherst, Massachusetts \\ 01003, and ${ }^{3}$ CAPES Foundation, Ministry of Education of Brazil, Brasília, DF 70040-020, Brazil
}

Vocal learning species must form and extensively hone associations between sounds and social contingencies. In songbirds, dopamine signaling guides song motor production, variability, and motivation, but it is unclear how dopamine regulates fundamental auditory associations for learning new sounds. We hypothesized that dopamine regulates learning in the auditory pallium, in part by interacting with local neuroestradiol signaling. Here, we show that zebra finch auditory neurons frequently coexpress D1 receptor (D1R) protein, neuroestradiol-synthase, GABA, and parvalbumin (PV). Auditory classical conditioning increased neuroplasticity gene induction in D1R-positive neurons. In vitro, D1R pharmacological activation reduced the amplitude of GABAergic and glutamatergic currents and increased the latter's frequency. In vivo, D1R activation reduced the firing of putative interneurons, increased the firing of putative excitatory neurons, and made both neuronal types unable to adapt to novel stimuli. Together, these findings support the hypothesis that dopamine acting via D1Rs modulates auditory association in the songbird sensory pallium.

Key words: auditory; neuromodulation; plasticity; songbird

Significance Statement

Our key finding is that auditory forebrain D1 receptors (D1Rs) modulate auditory plasticity, in support of the hypothesis that dopamine modulates the formation of associations between sounds and outcomes. Recent work in songbirds has identified roles for dopamine in driving reinforcement learning and motor variability in song production. This leaves open whether dopamine shapes the initial events that are critical for learning vocalizations, e.g., auditory learning. Our study begins to address this question in the songbird caudomedial nidopallium (NCM), an analog of the mammalian secondary auditory cortex. Our findings indicate that dopamine receptors are important modulators of excitatory/inhibitory balance and sound association learning mechanisms in the NCM, a system that could be a fundamental feature of vertebrate ascending auditory pathways.

\section{Introduction}

Studying vocal learning in songbirds continues to provide insight into the suite of mechanisms that support spoken language (Jarvis, 2019). The first step in the spoken language learning process is to make socially-contingent associations about complex sounds. This likely engages cortical brain structures, where sounds and meaning are bound.

\footnotetext{
Received Nov. 5, 2020; revised Mar. 9, 2021; accepted Apr. 21, 2021.

Author contributions: M.M.-L. and L.R.-H. designed research; M.M.-L. and H.M.B. performed research; L.R.-H. contributed unpublished reagents/analytic tools; M.M.-L. analyzed data; M.M.-L. wrote the paper.

This work was supported by the National Institutes of Health Grant R01NS082179 (L.R.-H.), the National Science Foundation Grant 10 S1354906 (L.R.-H., and H.M.B.), and the Coordenação de Aperfeiçoamento de Pessoal de Nivel Superior (CAPES; to M.M.-L.). We thank current and former members of the Healey Lab at the University of Massachusetts who helped with this project, especially Amanda Krentzel, Catherine deBournonville, Christina Moschetto, Daniel Pollak, Daniel Vahaba, Garrett Scarpa, Jeremy Spool, Katie Schroeder, Maaya Ikeda, Marcela Fernandez-Peters, and Rachel Frazier. We also thank Geng-Lin Li, Joseph Bergan, Jeffrey Podos, Karine Fenelon, and two anonymous reviewers for valuable input.

The authors declare no competing financial interests.

Correspondence should be addressed to Luke Remage-Healey at Iremageh@umass.edu.

https://doi.org/10.1523/JNEUROSCI.2823-20.2021

Copyright $\odot 2021$ the authors
}

Midbrain structures that process reinforcement can guide formation of auditory associations in the cortex through dopamine release. In mammals, dopamine in the primary auditory cortex drives auditory learning and plasticity of simple stimuli, such as pure tones (Bao et al., 2001; Schicknick et al., 2008, 2012). Dopaminergic signaling has also been examined in the songbird brain, but primarily in the context of reinforcement learning for song production and motivation (Leblois et al., 2010; Schmidt and Ding, 2014; Matheson and Sakata, 2015; Gadagkar et al., 2016; Tanaka et al., 2018). Much less is known about how dopamine signaling may regulate auditory processing and association of complex sounds such as song.

The songbird caudomedial nidopallium (NCM; Fig. $1 A$ ) is a pallial auditory region considered analogous to the center for speech comprehension in humans, Wernicke's area (Bolhuis et al., 2010). Neuronal responses in awake, restrained zebra finch NCM show stimulus-specific adaptation (SSA) to sounds played repeatedly, consistent with active memory formation (Chew et al., 1996; Lu and Vicario, 2017). This adaptation in NCM neurons reflects familiarity with songs, as well as song-consequence 

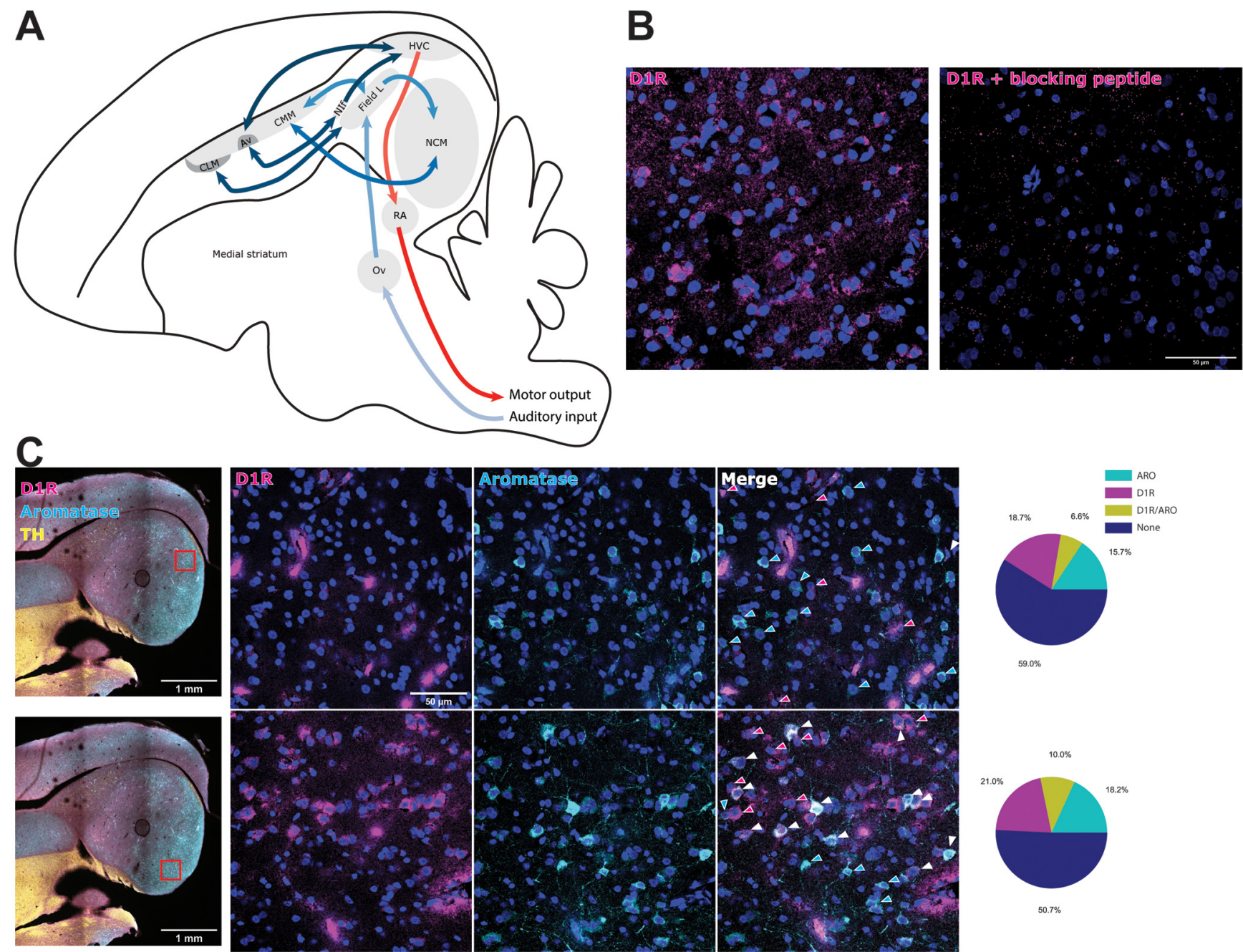

$50.7 \%$
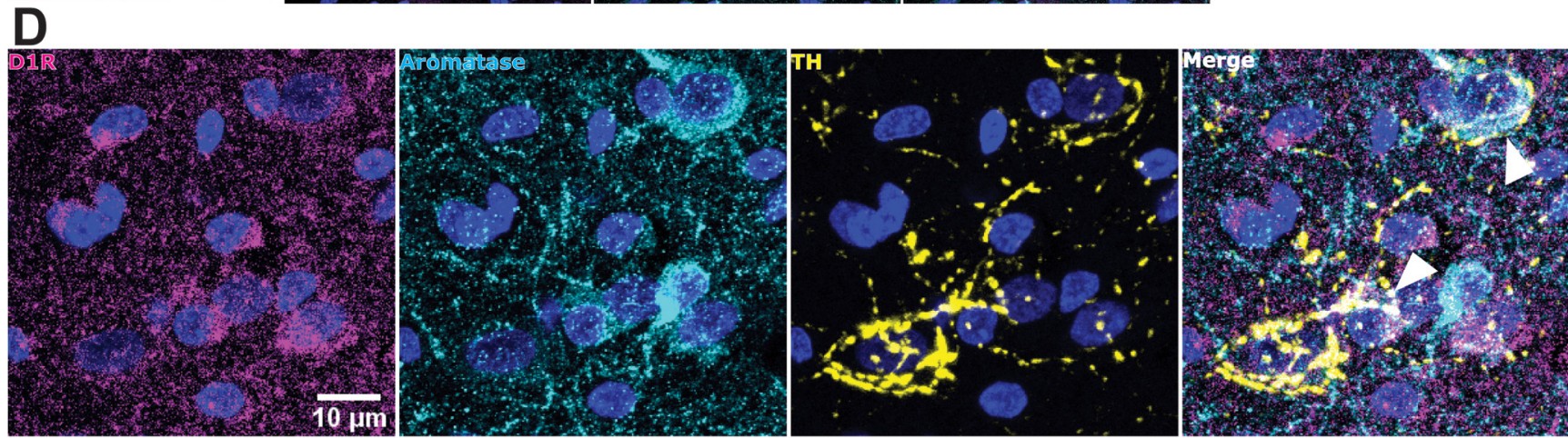

Figure 1. ARO and D1R proteins are coexpressed by NCM neurons. $\boldsymbol{A}$, Songbird auditory circuits. Lighter-to-darker colors illustrate the trajectory of auditory (blue) and motor (red) information. $\boldsymbol{B}$, Specificity confirmation of the anti-D1R antibody used here. Preincubation with blocking peptide virtually eliminated cellular staining. $\boldsymbol{C}$, Triple immunofluorescence staining for D1R, ARO, and TH (enzyme participating in dopamine synthesis). Magenta and cyan arrows indicate single-labeled D1R and ARO+ neurons, respectively. White arrows indicate double-labeling. $\mathbf{D}$, TH fibers are frequently found in close association with double-labeled neurons. Note that fibers tightly envelop double-labeled somata (arrows). Av, nucleus avalanche; AR0, aromatase; CMM, caudomedial mesopallium; D1R, dopamine D1 receptor; HVC, HVC (proper name); Ov, nucleus ovoidallis; NCM, caudomedial nidopallium; NIf, nucleus interfacialis; RA, robust nucleus of the arcopallium.

associations (Chew et al., 1996; Bell et al., 2015; Lu and Vicario, 2017). The NCM is an important target of a wide variety of neuromodulators, e.g., norepinephrine (Ikeda et al., 2015; Lee et al., 2018), neuroestradiol (Saldanha et al., 2000; Macedo-Lima and Remage-Healey, 2020), and dopamine (Matragrano et al., 2012). Moreover, NCM is rich in NMDA receptors (Saldanha et al., 2004), which are classically regarded as key players in cellular memory formation processes, such as long-term depression and potentiation (Lüscher and Malenka, 2012). NCM therefore is a highly plastic structure involved in the processing, and perhaps association, of complex sounds. However, the molecular and neuromodulatory mechanisms underlying associations between sound and context in higher-order circuits like NCM have not been elucidated.

Dopaminergic fibers permeate the secondary auditory regions NCM and the caudomedial mesopallium (CMM), but not the 
thalamo-recipient auditory region, Field L (Reiner et al., 1994; von Eugen et al., 2020). Dopamine receptor mRNA maps onto this architecture, such that D1 receptors (D1Rs) are abundant in $\mathrm{NCM}$ and CMM, and D2 receptors are abundant in CMM but not in NCM. Neither receptor is evident in the primary auditory pallium Field L (Kubikova et al., 2010). Neurons in the ventral tegmental area have been reported to project to the NCM and could provide a learning-contingent source of dopamine to this region (Yanagihara et al., 2019). Indeed, hearing song increases rapid turnover of dopamine in songbird auditory regions, especially in the NCM (Matragrano et al., 2012). Therefore, dopamine signaling is a feature distinguishing secondary from primary auditory pallium in songbirds, providing an anatomic circuit-basis for dopamine-dependent auditory learning. The extent to which dopamine signaling modulates the encoding and processing of stimuli in songbird neurons, however, is unclear.

In this study, we hypothesized that dopamine signaling via D1Rs mediates NCM neural circuit plasticity. We obtained anatomic, behavioral, and physiological evidence that support this hypothesis. First, we used immunofluorescence to characterize D1R-expressing neurons regarding aromatase (ARO), GABA, and parvalbumin (PV) expression. To investigate whether D1Rs were involved in auditory association, we examined the expression of the immediate-early gene early-growth response (EGR)1 in D1R-expressing neurons during an auditory classical conditioning task. To characterize the synaptic effects of D1R activation we employed in vitro intracellular recordings and recorded glutamate and GABAergic currents while delivering a D1R agonist. Finally, to examine the functional effects of D1R activation on auditory physiology and plasticity we delivered the D1R agonist during in vivo awake extracellular multielectrode recordings and evaluated whether D1R activation modulated neuronal firing patterns and SSA in response to auditory signals.

\section{Materials and Methods}

\section{Animals}

A total of 53 adult ( $>90 \mathrm{~d}$ old) zebra finches ( 34 males) were used across all experiments. Birds came from the University of Massachusetts Amherst colony (14/10 h light/dark cycle) and were not actively breeding (single-sex cages). All procedures were in accordance with the Institutional Animal Care and Use Committee at the University of Massachusetts Amherst.

\section{Immunofluorescence, imaging, and quantification}

Four males and four females were used for immunofluorescence experiments, to characterize the phenotype of D1R + cells. Briefly, animals were taken from the aviary, deeply anesthetized, and perfused with icecold PBS followed by room-temperature phosphate-buffered $4 \%$ paraformaldehyde (PFA). Brains were extracted, postfixed in the same fixative overnight, cryoprotected in $30 \%$ sucrose and frozen until processing. With a cryostat, $40-\mu \mathrm{m}$ parasagittal sections were made and sampled in four subseries collected in cryoprotectant solution and stored at $-20^{\circ} \mathrm{C}$ until processed.

Of the above subjects, tissue from two males and two females was processed for D1R, ARO, and tyrosine hydroxylase (TH) triple immunofluorescence; tissue from all four males and four females was processed for D1R, GABA, and PV triple immunofluorescence.

Sections containing NCM were selected and transferred from cryoprotectant to phosphate buffer $(\mathrm{PB})$ and washed $3 \times 15 \mathrm{~min}$. They were then incubated in 10\% normal goat serum (NGS; Vector Labs) in $0.3 \%$ Triton X-100 (Thermo Fisher Scientific) in PB (PBT) for $2 \mathrm{~h}$. Primary antibody solutions (Table 1) were prepared in $10 \%$ NGS in $0.3 \%$ PBT. To confirm antibody specificity, in a subset of sections the D1R antibody was preincubated for $1 \mathrm{~h}$ with blocking peptide (Fig. 1B). Sections were incubated with primary antibodies for $1 \mathrm{~h}$ at room temperature, followed
Table 1. Primary antibody table

\begin{tabular}{lllll}
\hline Antibody & Type & Host & Dilution & Company \\
\hline Anti-D1DR & Polyclonal & Guinea pig & $1: 100$ & Alomone Labs \\
D1DR peptide & - & Rat & $1: 10$ & Alomone Labs \\
Anti-AR0 & Polyclonal & Rabbit & $1: 2000$ & Azac \\
Anti-AR0 & Polyclonal & Sheep & $1: 2000$ & Bethyl Labs \\
Anti-EGR1 & Polyclonal & Rabbit & $1: 2000$ & Santa Cruz \\
Anti-TH & Monoclonal & Mouse & $1: 2000$ & Immunostar \\
Anti-GABA & Polyclonal & Rabbit & $1: 1000$ & Sigma \\
Anti-PV & Monoclonal & Mouse & $1: 10000$ & Millipore \\
\hline
\end{tabular}

by $2 \mathrm{~d}$ at $4^{\circ} \mathrm{C}$. Then, sections were washed $3 \times 15 \mathrm{~min}$ in $0.1 \%$ PBT and transferred to secondary antibody solutions (goat polyclonals; Thermo Fisher Scientific; 1:200) prepared in 0.3\% PBT. Finally, sections were washed in $3 \times 10 \mathrm{~min}$ in $0.1 \%$ PBT and kept in the same solution in the fridge until mounted ( 1 or $2 \mathrm{~d}$ later) and coverslipped with ProLong Diamond with DAPI (Thermo Fisher Scientific).

Images were taken with a confocal microscope (Nikon A1si). First, NCM was localized and a $4 \times 4$ large image was taken at $10 \times$ magnification. Then, using only the DAPI channel, the microscope stage was digitally controlled and moved to selected locations on the $10 \times$ images, at the ventral and dorsal posterior edges of NCM (Fig. 1C). Then, $15 \mu \mathrm{m}$ (1- $\mu \mathrm{m}$ step size) $\mathrm{z}$-stack images were taken at $60 \times$ magnification, starting from the top-most surface of the section. Two sections per hemisphere per animal were imaged. All laser intensities were maintained uniform across all images within experiments.

D1R antibody penetration noticeably decayed at $\sim 5 \mu \mathrm{m}$ deep into the tissue, therefore only the top $5 \mu \mathrm{m}$ of each $\mathrm{z}$-stack was quantified. Cell counts were performed by a blinded experimenter using Fiji (ImageJ; NIH). Briefly, color histograms were set individually for each image so that background was predominantly dark and only strong signals were counted. Only antibody localization around the nuclei (DAPI) was included. Antibody quantification was done using the z-stack, while DAPI quantification was done using the $\mathrm{z}$-max-projection image.

Because DAPI is a universal nuclear stain, only cells with large, ovoidal nuclei (presumably neurons) were counted. The antibody stains employed here further increase this confidence because cytoplasm shape and occasionally dendrites were evident. Still, this method likely underestimates the antibody positivity rate, since glial nuclei are more likely to be overrepresented in the DAPI counts. Of note, ARO protein has been observed in radial glia in songbirds and other species (Alvarez-Buylla et al., 1987; Forlano et al., 2006).

\section{Classical conditioning and immediate early gene expression}

Stimuli and behavioral procedure

Twenty adult zebra finches (10 males) were used in this experiment. Animals were individually isolated for $\sim 17.5 \mathrm{~h}$ in a sound attenuation chamber. The cage set up consisted of three horizontal perches evenly spread out parallel to the cage floor (Fig. 2C). Food and water were located near the middle perch. An LCD monitor and a speaker were positioned adjacent to the cage. Behavior was recorded with a camera positioned on the opposite side of the cage from the monitor.

Animals were exposed to two Experimental conditions (paired vs unpaired) and two reference conditions (song vs silence). The aim of the Experimental conditions was to test whether auditory-visual classical conditioning would induce EGR1 expression. Silent videos of conspecifics elicit behavioral attention and engagement and can be employed as social reinforcers for zebra finches (Galoch and Bischof, 2007). Furthermore, neither silent videos nor tones are strong drivers of EGR1 expression in NCM, which confers a dynamic range to evaluate the effects of stimuli combination (Avey et al., 2005; Sanford et al., 2010). Thus, in the paired condition (three males, three females), animals were exposed to repetitions of a 2 -s pure tone $(2 \mathrm{kHz} ; \sim 65 \mathrm{~dB})$, the conditioned stimulus, followed by a 2-s delay then a 6-s silent video of a male zebra finch singing ( 6 different video clips were randomized), the unconditioned stimulus. Stimuli were presented 30 times (30- to 90-s interstimulus interval) over a 30-min window. In the unpaired condition (three 

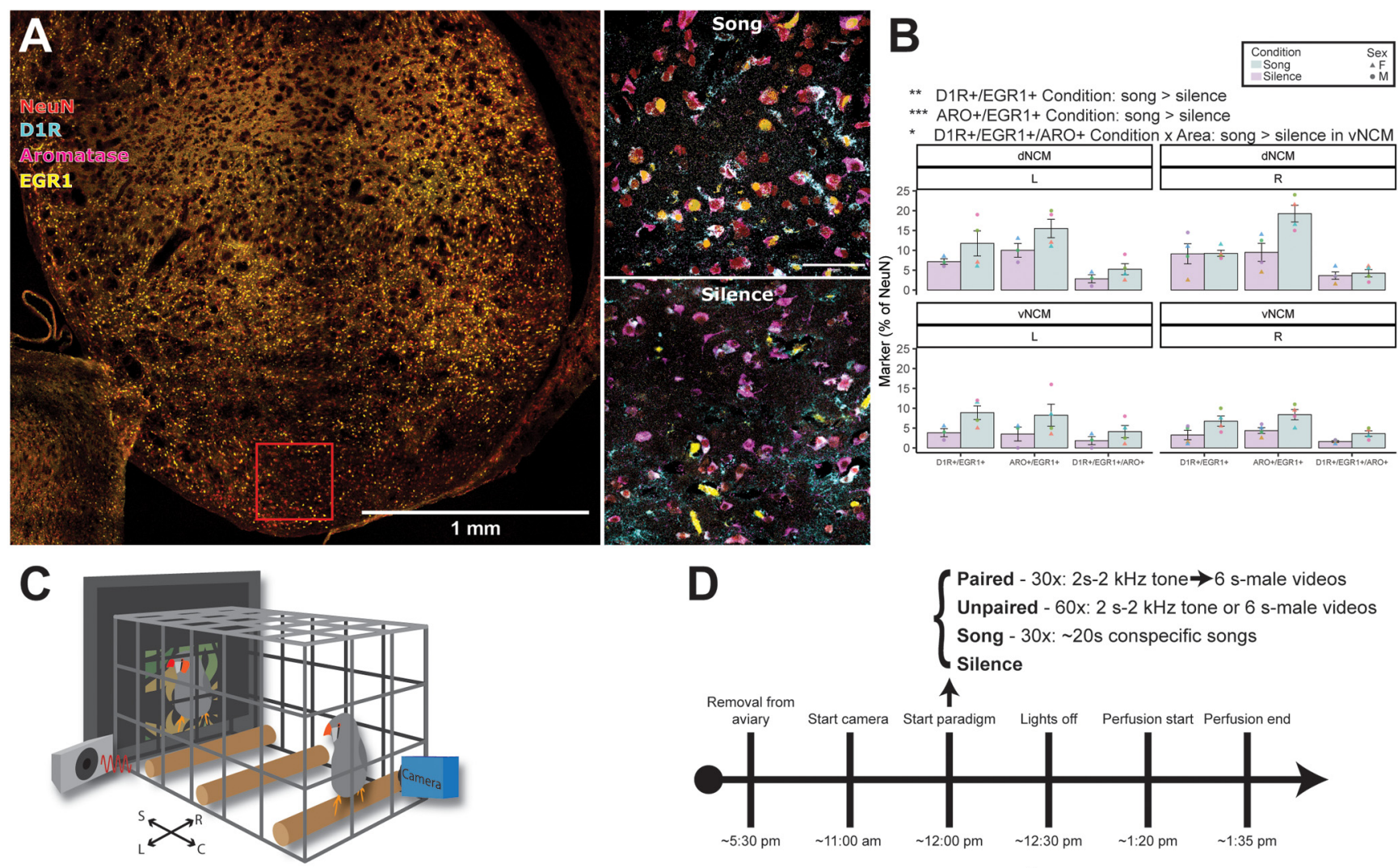
Paired - 30x: 2s-2 kHz tone $\rightarrow 6$ s-male videos Unpaired - 60x: $2 \mathrm{~s}-2 \mathrm{kHz}$ tone or $6 \mathrm{~s}$-male videos Song - 30x: $\sim 20$ s conspecific songs
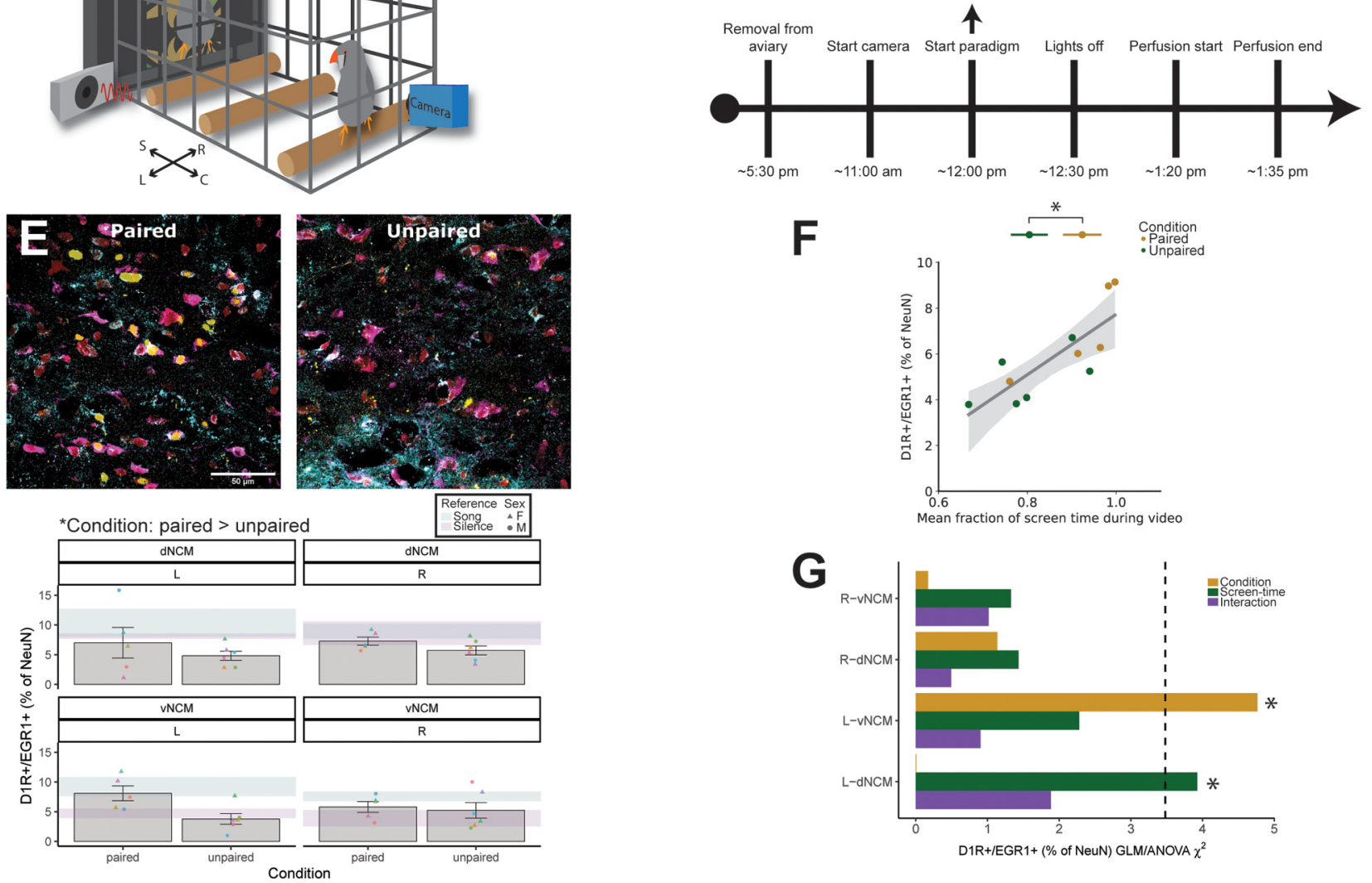

Figure 2. EGR1 protein expression in NCM neurons after song stimulation alone or auditory classical conditioning with pure tone and social reinforcement. $\boldsymbol{A}, \boldsymbol{B}$, Song stimulation increases EGR1 expression in D1R + and ARO + across areas and hemispheres and D1R+/ARO + neurons in the vNCM across hemispheres as compared with Silence. C, Classical conditioning behavioral apparatus and (D) timeline. After behavioral manipulations, a 50-min lights-off period followed, then animals were quickly perfused and brains were processed for EGR1, D1R, and ARO labeling $(\boldsymbol{A}, \boldsymbol{E}) . \boldsymbol{E}, \mathrm{D} 1 \mathrm{R}+/ \mathrm{EGR} 1+$ neurons are higher in the paired than in the unpaired group. Note that in left ventral NCM the paired group mean is within the range of the song reference group. $\boldsymbol{F}$, Screen-time predicts EGR1 expression in D1R+ neurons and is higher in paired than in unpaired groups. G, GLM/ANOVA $\chi^{2}$ results for the frequency of D1R+/EGR1+ neurons after classical conditioning paradigm within each hemisphere $\times$ area combination, using condition and screen-time as predictors. Significant effects were found for condition and not screen-time in the LvNCM, and of screen-time and not condition in the L-dNCM (paired $>$ unpaired in both); ${ }^{*} p<0.05,{ }^{* *} p<0.01,{ }^{* * *} p<0.001$; L, left; R, right; NCM, caudomedial nidopallium; dNCM, dorsal NCM; vNCM, ventral NCM. Marker colors represent data from individual subjects within each experiment.

males, three females), animals were presented with the same amount of stimuli (30 videos and 30 tones), but those were presented separately in pseudorandom order (15- to 45-s interstimulus interval) over a 30-min window. Videos were scaled so that birds in the video were displayed at approximately real sizes. Conspecific song playback after periods of isolation drive robust expression of EGR1 in NCM when compared with silence conditions (Mello and Clayton, 1994; Mello et al., 1992). Therefore, we exposed birds to reference conditions to provide expression level references to our experimental groups. In the song condition, animals were exposed to three different conspecific bouts of songs (each 
18-21 s long; $\sim 65 \mathrm{~dB}$ ) repeated 10 times in pseudorandom order over $30 \mathrm{~min}$. In the silence condition, animals were not presented with any stimulus for $30 \mathrm{~min}$.

\section{Immunofluorescence}

EGR1 protein expression in NCM peaks between 1 and $2 \mathrm{~h}$ after induction (Mello and Ribeiro, 1998). Therefore, in all conditions, after the presentation of the last stimulus (or after $30 \mathrm{~min}$ in the Silence group), chamber lights were turned off (to minimize further stimulation) and animals remained in the chamber for an additional $50 \mathrm{~min}$ before they were retrieved for perfusion $(\sim 10 \mathrm{~min}$ until PFA). Total time from beginning of exposure to fixative exposure was $\sim 1.5 \mathrm{~h}$.

After perfusion, brains were processed, sectioned, and stained as described above, but with the following differences. A triple immunofluorescence protocol was performed using antibodies against NeuN, D1R, EGR1, and ARO (Table 1) prepared in 10\% donkey serum (Vector Labs) in $0.3 \%$ PBT. Donkey polyclonal secondary antibodies (Thermo Fisher Scientific; 1:200) were used. Imaging and cell counts were also performed as described above, except NeuN was used as a background stain.

\section{Behavioral scoring and analyses}

Video recordings were snipped into 15-s clips around stimulus presentations using custom Python code. Only the experimental conditions (paired and unpaired) were analyzed.

Clips were scored twice, once for state and once for event behaviors. State behaviors included beak direction (left, right, pointed at screen, or pointed at camera), sleeping, eating, drinking or continuously moving/ flying. Event behaviors were not mutually exclusive with states, and included vocalization, singing, feather ruffling, head tilts, hopping, and gaping. Videos were scored by an experimenter blinded to the subject ID and trial order. Experimental groups could be inferred by the observer because in the paired group, the video playback shortly followed the tone presentation. Behaviors were scored using JWatcher (Blumstein and Daniel, 2007).

Behavioral data were extracted and processed using custom Python scripts. Timestamps and continuous behavior durations were aligned to stimulus timestamps and quantified as rates ( $\mathrm{Hz}$; event behaviors) or percent of time spent (fraction of stimulus duration; state behaviors). Behaviors outside of the stimuli presentation windows were not analyzed. Beak direction behaviors that enabled subjects to see the screen (beak pointed left, right and toward screen) were summed to comprise a new category termed "screen-time." Conversely, beak direction opposite to the screen (back of the head facing video), eating, drinking and sleeping were averaged to comprise a category termed "distractibility."

\section{In vitro whole-cell patch clamp}

Recordings

Sixteen males were used for slice recordings across two experiments. We focused these experiments on males to further explore mechanisms proposed in a previous behavioral study done in males (Macedo-Lima and Remage-Healey, 2020). We note that we did not observe systematic sex differences in the immunofluorescence and in vivo electrophysiology findings, but we do not discard the possibility of sex differences.

After swift decapitation, the top of the skull was resected and the head was immediately immersed in a Petri dish filled with ice-cold carbogen-aerated cutting solution $\left(0-\mathrm{Mg}^{2+}\right.$ cutting solution: $222 \mathrm{~mm}$ glycerol, $25 \mathrm{~mm} \mathrm{NaHCO}_{3}, 2.5 \mathrm{~mm} \mathrm{KCl}, 1.25 \mathrm{~mm} \mathrm{NaH}_{2} \mathrm{PO}_{4}, 0.5 \mathrm{~mm} \mathrm{CaCl}, 34$ $\mathrm{mm}$ glucose, $0.4 \mathrm{~mm}$ ascorbic acid, $2 \mathrm{~mm} \mathrm{Na}$-pyruvate, and $3 \mathrm{~mm}$ myoinositol; standard cutting solution: same as above except $25 \mathrm{~mm}$ glucose and $3 \mathrm{~mm} \mathrm{MgCl}_{2} ; \sim 320 \mathrm{mOsm} / \mathrm{kg}$, pH 7.4). In the Petri dish, the cerebellum was resected, and the brain was removed from the skull. Then, the brain was removed from the cutting solution and placed on an icecold Petri dish lid covered with Kimwipe (Kimberly-Clark Professional). The lateral forebrain of both hemispheres was cut parasagittally to yield flat lateral surfaces, and cerebral hemispheres were bisected. The lateral edges of each hemisphere were then dabbed dry on the Kimwipe, glued (cyanoacrylate) to the cutting stage and immediately immersed in the vibratome (VT1000S, Leica Biosystems) chamber filled with ice-cold carbogen-aerated cutting solution. Slices were cut at 250 or $300 \mu \mathrm{m}$ starting from the medial edge which contains NCM. NCM does not have clearly defined lateral boundaries, but song-inducible gene expression experiments indicate strong responses that extend $\sim 1 \mathrm{~mm}$ from the midline (Mello and Clayton, 1994), thus only the first three sections $(\sim 750-900 \mu \mathrm{m})$ were used for recordings. After cutting, slices were transferred to $37^{\circ} \mathrm{C}$ carbogen-aerated $0-\mathrm{Mg}^{2+}$ or standard recording solution $\left(0-\mathrm{Mg}^{2+}\right.$ : $111 \mathrm{~mm} \mathrm{NaCl}, 25 \mathrm{~mm} \mathrm{NaHCO}, 2.5 \mathrm{~mm} \mathrm{KCl}, 1.25 \mathrm{~mm}$ $\mathrm{NaH}_{2} \mathrm{PO}_{4}, 2 \mathrm{~mm} \mathrm{CaCl}$, $28 \mathrm{~mm}$ glucose, $0.4 \mathrm{~mm}$ ascorbic acid, $2 \mathrm{~mm} \mathrm{Na}$ pyruvate, and $3 \mathrm{~mm}$ myo-inositol; standard: idem except $25 \mathrm{~mm}$ glucose and $3 \mathrm{~mm} \mathrm{MgCl} ; \sim 320 \mathrm{mOsm} / \mathrm{kg}$, pH 7.4). After a 30 -min recovery at $37^{\circ} \mathrm{C}$ and a $30 \mathrm{~min}$ stabilization at room temperature, recordings started. All recordings were performed at room temperature. Slices from both hemispheres were used. Recording location within NCM (e.g., ventral vs dorsal) was not carefully noted, but most recordings were done in the ventral NCM. Dorsal NCM cells were only used if ventral NCM cells were not feasible to patch.

Recording pipettes (borosilicate glass) were pulled with a vertical pipette puller (PC-10, Narishige International) and had a tip resistance of 4-7 M $\Omega$ when submerged in the recording solution and backfilled with either K-gluconate-based or CsCl-based solution, for EPSC and IPSC recordings, respectively (K-gluconate-based: $\sim 95 \mathrm{~mm}$ K-gluconate, 20 mм KCl, $0.1 \mathrm{~mm} \mathrm{CaCl}_{2}$, 5 mм HEPES, 5 mм EGTA, 3 mм MgATP, 0.5 $\mathrm{mm} \mathrm{NaGTP}$, and $20 \mathrm{~mm}$ creatine-phosphate disodium; osmolarity adjusted to $\sim 295 \mathrm{mOsm} / \mathrm{kg}$ with K-gluconate; $\mathrm{pH}$ 7.4; CsCl-based: $\sim 120 \mathrm{~mm} \mathrm{CsCl}, 8 \mathrm{~mm} \mathrm{NaCl}, 10 \mathrm{~mm}$ TEA-Cl, 0.2 mм EGTA, $10 \mathrm{~mm}$ HEPES, $2 \mathrm{~mm}$ MgATP, and $0.2 \mathrm{~mm}$ NaGTP; osmolarity adjusted to $\sim 295 \mathrm{mOsm} / \mathrm{kg}$ with $\mathrm{CsCl} ; \mathrm{pH}$ 7.4). Internal solutions also contained $0.1 \%$ Alexa Fluor 488-hydrazide (Thermo Fisher Scientific) and $0.1 \%$ Neurobiotin (Vector Labs) for "online" and post hoc detection (see below) of the cell, respectively.

Cells were identified with an inverted microscope (Eclipse FN1, Nikon) with DIC optics. Recordings were made inside a Faraday cage using an EPC-10 amplifier and recorded and compensated (series resistance, slow/fast capacitance) with PatchMaster software (HEKA). Liquid junction potential was automatically subtracted. Traces were digitized at $20 \mathrm{kHz}$. Recordings were made in voltage clamp mode at $-70 \mathrm{mV}$. After whole-cell configuration was achieved, cells were allowed to stabilize for 5 min. Then, baseline drug cocktails (bicuculline or DNQX) were delivered and allowed to act for a minimum of $2 \mathrm{~min}$. Drug-containing solutions were gravity-delivered and flow-matched to a $\sim 2 \mathrm{ml} / \mathrm{min}$ peristaltic pump (Cole-Palmer MasterFlex L/S) outtake. Tissue chamber capacity was $\sim 1 \mathrm{ml}$. Recording quality was constantly monitored between recording blocks and recordings were aborted if series resistance rose above $40 \mathrm{M} \Omega$.

Ten males were used for AMPA/kainate/NMDA EPSCs (sEPSCs) recordings. For sEPSC isolation, bicuculline $(20 \mu \mathrm{M}$; Santa Cruz) was added to the $0-\mathrm{Mg}^{2+}$ recording solution. For some recordings, after bicuculline was delivered and allowed to take effect, a 1-min baseline recording was made, but for 11 out of 18 cells, a 7 -min rundown recording followed. Then, \pm -SKF-38393 hydrochloride (10 or $50 \mu \mathrm{M}$; Abcam; Ding and Perkel, 2002) was delivered and a 7 min recording was timed to the start of the delivery. Maximum concentration of drug is estimated to have been achieved within $1 \mathrm{~min}$. Finally, a 10-min washout recording was timed to the start of SKF-38393 clearance, and complete washout is estimated to have been achieved within $1 \mathrm{~min}$. Finally, to confirm the nature of the currents, D-AP-5 (50 $\mu \mathrm{M}$; Abcam or Alomone) was delivered and currents were monitored for $\sim 5 \mathrm{~min}$.

Six males were used for spontaneous GABA IPSCs (sIPSCs) recordings. For sIPSC isolation, DNQX ( $20 \mu \mathrm{M}$; Tocris) was added to the standard recording solution. Recordings were made similarly to EPSC recordings, except different cells were used for SKF $(n=7)$ and rundown experiments $(n=4)$. After a 1-min baseline recording, either SKF-38393 $(10 \mu \mathrm{M})$ or nothing (rundown) was added to the recording solution and currents were monitored for $7 \mathrm{~min}$. Then, a 10-min washout (or continued rundown) followed. Finally, to confirm the nature of the currents, bicuculline $(20 \mu \mathrm{M})$ was added to the recording solution and currents were monitored for $\sim 5 \mathrm{~min}$.

After recordings were completed, the recording pipette was slowly retrieved, and slices were drop-fixed overnight in $4 \%$ PFA in PB. Then, 
they were transferred to cryoprotectant solution and kept at $-20^{\circ} \mathrm{C}$ until processed.

\section{Analyses}

Recordings were analyzed in IgorPro 6 (WaveMetrics). All traces were downsampled $(5 \times)$ and lowpass filtered at $500 \mathrm{~Hz}$.

In sEPSC experiments, for amplitude measurements $[n=9$ from four animals; four from the left (three animals) and 5 cells from the right (three animals) hemispheres], cells were only included in the analysis if series resistance did not change by $>20 \%$ from baseline values. For frequency recordings, all cells $[n=18$ from 10 animals; 6 cells from the left (5 animals), 10 cells from the right (8 animals) hemisphere and 2 unnoted cells ( 2 animals)] were analyzed, as recording quality fluctuations are not expected to interfere with their detection, because of their high amplitude ( $>50 \mathrm{pA}$; noise band $\sim 5 \mathrm{pA}$ ). Currents were thresholded and manually curated with NeuroMatic (Rothman and Silver, 2018). After curation, currents were automatically measured by custom IgorPro code.

For sIPSC recordings, cells whose series resistance changed $>20 \%$ from baseline were excluded from all analyses. We obtained stable recordings of 11 cells (six animals), seven of which (from five animals) received the SKF treatment. Those consisted of two cells from the left (two animals) and five from the right hemisphere (five animals). For each cell, one template current was manually selected and spontaneous sIPSCs were automatically detected using a spontaneous current detection algorithm (Clements and Bekkers, 1997) implemented by Geng-Lin Li for IgorPro. After detection, all sIPSCs were measured automatically by custom IgorPro code.

For imaging cells containing neurobiotin, slices were washed $3 \times 15$ min in PB and incubated in 10\% NGS in $0.3 \%$ PBT for $2 \mathrm{~h}$. Sections were then incubated with rabbit anti-ARO diluted 1:2000 in 10\% NGS in $0.3 \% \mathrm{PBT}$ for $1 \mathrm{~h}$ at room temperature, followed by $2 \mathrm{~d}$ at $4^{\circ} \mathrm{C}$. Then, sections were washed $3 \times 15 \mathrm{~min}$ in $0.1 \% \mathrm{PBT}$ and transferred to $0.3 \% \mathrm{PBT}$ containing goat anti-rabbit at $1: 200$ and Streptavidin-DyLight- 488 (Vector) at 1:200. Finally, sections were washed in $3 \times 10 \mathrm{~min}$ in $0.1 \%$ PBT and kept in the same solution in the fridge until mounted $(1$ or $2 \mathrm{~d}$ later) and coverslipped with ProLong Diamond with DAPI (Thermo Fisher Scientific). Cells were imaged using a confocal microscope (Nikon A1si) at $20 \times$ and $100 \times$ magnification.

\section{In vivo awake head-fixed electrophysiology}

\section{Headpost implantation and craniotomy surgery}

Five males and four females were retrieved from the single-sex cages and implanted with headposts. Briefly, under isoflurane anesthesia, custommade headposts were lowered on the top of the beak and secured with dental cement. Skull markings over NCM (1.1 lateral, 1.4 anterior from the bifurcation of the mid-sagittal sinus, $45^{\circ}$ head angle) were performed, large craniotomies over NCM were made and meninges were resected. During the recordings (described below), electrode bundles were always inserted medio-caudally to markings (within 0.5 lateral, 1.2 anterior from mid-sagittal sinus). A small craniotomy was made in an anterolateral part of the skull for the implantation of a silver ground wire using cyanoacrylate. Craniotomies were sealed with Kwik-Cast (World Precision Instruments), and birds were allowed to recover from anesthesia. Recordings were performed within $4 \mathrm{~d}$ of surgery.

\section{Retrodialysis-microdrive (RetroDrive) fabrication}

Custom retrodialysis probe-coupled multielectrode drives (RetroDrives; Fig. 6) were assembled in house. RetroDrives consisted of a circular printed circuit board (PCB; Sunstone Circuits) soldered to a 36-pin connector (A79026-001, Omnetics), and a 5-mm 17-G guide tube (stainless steel; Component Supply Company). A strand of $28 \mathrm{G}$ enameled copper magnet wire (Remington Industries) was soldered to the PCB ground. Three polyimide tubes $(2 \times 198 \mu \mathrm{m} ; 1 \times 100 \mu \mathrm{m}$ in diameter $)$ were inserted through the guide tube, glued side-by-side on the wall of the guide tube and cut to be flush with the guide tube. Tetrodes were made by twice-folding and twisting polyimide-coated $\mathrm{NiCr}$ tetrode wire (Sandvik) with a tetrode spinner (LabMaker), followed by brief low-heat low-air treatment with a heat gun $(\mathrm{CO}-\mathrm{Z} 858 \mathrm{D})$ to join wires. Tetrodes were inserted through the larger polyimide tubes (four in each) and pinned to the PCB. A single reference wire $(50 \mu \mathrm{m}$ polyimide-coated $\mathrm{NiCr}$ wire; Sandvik) was inserted through the smaller polyimide tube and pinned to the PCB. Wires were glued to the top of the polyimide tubes. A microdialysis cannula (CMA8011085; Harvard Apparatus) was glued adjacent to the polyimide tubes containing tetrodes, such that a minimum of $3 \mathrm{~mm}$ of cannula protruded from the guide tube. Tetrodes were cut with precision scissors $(14058-11$, FST) to $\sim 0.5 \mathrm{~mm}$ from the tip of the cannula. The reference wire was cut to a similar length at an acute angle. When $1 \mathrm{~mm}$ membrane probes (CMA8011081; Harvard Apparatus) were inserted, the tips of the probe and wires were offset by $\sim 0.5 \mathrm{~mm}$. Importantly, the horizontal distance between probe wires were $\sim 0.2 \mathrm{~mm}$. Finally, tetrodes were gold-plated to $200-250 \mathrm{k} \Omega$ impedance and all wires and pins were covered with liquid electrical tape (Gardner Bender) and allowed to dry. RetroDrives were confirmed to successfully operate in NCM using baclofen/muscimol delivery to locally silence neurons within minutes in an earlier study (Macedo-Lima and Remage-Healey, 2020).

\section{Recording protocol}

On the day of the recording, a microdialysis probe was perfused with artificial CSF (aCSF; described below) using a microinjection pump (PHD2000, Harvard Apparatus). RetroDrive wires were dipped in 6.25\% DiI (Thermo Fisher Scientific) in $100 \%$ ethanol for visualization of electrode tracks. Then, the animal was comfortably restrained and headfixed. The Kwik-Cast was removed from the craniotomy over one of the hemispheres. Animal and RetroDrive grounds were connected using alligator clips. The microdialysis probe was inserted through the cannula and the RetroDrive was lowered to NCM $(\sim 1.5-2 \mathrm{~mm}$ from brain surface; mediocaudally to skull markings). Importantly, tetrodes were positioned medially to the probe such that wires were $\sim 0.5 \mathrm{~mm}$ lateral and $\sim 1.2$ anterior from the stereotaxic zero (midsagittal sinus).

Recordings were made while animals listened to auditory stimuli and aCSF (PRE) followed by SKF-38393 (SKF) followed by aCSF (POST) were infused during song playback to assess within-subject the effects of SKF on responses to auditory stimuli (described in detail below). Recordings were completed within $4 \mathrm{~h}$ of restraint.

Recordings were made from both hemispheres in different days. When recording in the first hemisphere was completed, the craniotomy was resealed with Kwik-Cast and the animal was returned to the home cage. Within $2 \mathrm{~d}$, the second hemisphere recording was made, after which the animal was overdosed with isoflurane and decapitated. The brain was drop-fixed and cryoprotected in $30 \%$ sucrose in $10 \%$ formalin, and frozen until cutting. Cryostat sections were obtained at $40 \mu \mathrm{m}$ and imaged to confirm location of wires and probe.

Recordings were amplified and digitized by a 32-channel amplifier and evaluation board (RHD2000 series; Intan Technologies) and sampled at $30 \mathrm{kHz}$ using Intan or Open Ephys software. An Arduino Uno (Arduino) was connected to the recording computer to deliver TTL pulses to the evaluation board's DAC channel bracketing the beginning and end of the audio stimuli (described below) to optimize detection during analysis. Audio playback and TTL pulses were controlled by a custom-made MATLAB (MathWorks) script which also controlled the Arduino and sent a copy of the audio analog signal to the evaluation board ADC channel.

Stimuli

Zebra finch songs were obtained from multiple databases (http://ofer.sci. ccny.cuny.edu/song_database), therefore unlikely to have been familiar to our subjects. Twenty-four song files from unrelated birds were bandpass filtered at $0.5-15 \mathrm{kHz}$ and trimmed to include two consecutive motifs without introductory notes in Adobe Audition (Adobe) and mean amplitude-normalized to $70 \mathrm{~dB}$ in Praat (Boersma and van Heuven, 2001). Songs were randomly and equally split into two sets, then into three subsets containing four songs each. For each animal, 1 set was used per hemisphere and, within a hemisphere recording, 1 subset was used per treatment. This was done to ensure that for each treatment birds listened to novel stimuli, because NCM neurons exhibit SSA (Chew et al., 1996). Importantly, there was no difference in neuronal 
firing rates to different subsets, controlled by each stimulus and within each subject and each neuron [generalized linear modeling (GLM)/ ANOVA: subset: $\chi^{2}(5)=5.173, p=0.395$ ]. Therefore, responses across treatments are comparable as they were presumed to reflect responses to novel stimuli.

Each playback session consisted of four conspecific songs, repeated 30 times each in pseudorandomized order. Interstimulus interval was pseudorandom within the interval $5 \pm 2 \mathrm{~s}$. The speaker was positioned $\sim 30 \mathrm{~cm}$ from the animal. Sound level was amplified to $\sim 65 \mathrm{~dB}$ as measured by a sound level meter at the animal's position (RadioShack). Playback trial duration lasted $\sim 20 \mathrm{~min}$. Recordings were performed inside a Faraday cage in a quiet room with constant low background noise from a ceiling vent.

Recordings were made from each hemisphere on different days. For each animal's first recording, the starting hemisphere was randomized in the first subjects, then counterbalanced between sexes. The stimulus set was also initially randomized, then counterbalanced across sexes and hemispheres, but the subset selected for each treatment was always randomized.

\section{Drugs and treatment}

SKF-38393 (Abcam) aliquots were made in double-distilled water (20 mM; $10 \mu$ l; Schicknick et al., 2012) and kept at $-20^{\circ} \mathrm{C}$. On the day of the experiment, one aliquot was added to $1 \mathrm{ml}$ (final concentration $0.2 \mathrm{mM}$ as in Schicknick et al., 2012) of previously frozen aCSF aliquots (199 mM $\mathrm{NaCl}, 26.2 \mathrm{~mm} \mathrm{NaHCO}$, $2.5 \mathrm{~mm} \mathrm{KCl}, 1 \mathrm{~mm} \mathrm{NaH} \mathrm{PO}_{4}, 1.3 \mathrm{~mm} \mathrm{MgSO}$, $2.5 \mathrm{mM} \mathrm{CaCl}_{2}, 11 \mathrm{~mm}$ glucose, and $0.15 \mathrm{~mm}$ bovine serum albumin; $\mathrm{pH}$ 7.4). All aliquots were filtered before being loaded into the RetroDrive. Treatment and playback timeline are described in Figure 6. After each round of playback, treatment syringes were switched and a 30-min infusion period elapsed. Retrodialysis speed was set to $2 \mu \mathrm{l} / \mathrm{min}$ (as in Remage-Healey et al., 2010; Remage-Healey and Joshi, 2012; Vahaba et al., 2017).

\section{Analyses}

Sound playback timestamps were detected using a custom-made audio convolution algorithm in MATLAB.

Recordings were highpass filtered at $300 \mathrm{~Hz}$ and common-median filtered in MATLAB (MathWorks). Single-unit sorting was done with Kilosort (Pachitariu et al., 2016). Sorting results were manually curated in Phy (https://github.com/cortex-lab/phy) and only well-isolated units were used (high signal-to-noise ratio; low violation of refractory period (the number of interspike intervals within a $1 \mathrm{~ms}$ refractory period was $0.25 \%$ median in our units), low contamination with other units; segregation in waveform PCA space).

After sorting, for each single-unit, 2000 waveforms were selected pseudorandomly and measured (peak-to-peak duration and ratio; Fig. $4 A$ ) in MATLAB. All further data processing was done in Python and R.

Spontaneous firing rates were calculated using $500 \mathrm{~ms}$ preceding each stimulus playback trial. Within each treatment condition, spontaneous and stimulus firing rates were averaged across stimuli and trials. Peristimulus time histograms (PSTHs) were generated using 10-ms time bins.

$Z$ scores were calculated by the formula $Z=$ $\frac{\operatorname{Mean}(S)-\operatorname{Mean}(B)}{\sqrt{\operatorname{Var}(S)+\operatorname{Var}(B)-2(\operatorname{Cov}(S, B))}}$, where S and B are the stimulus and spontaneous firing rates across stimulus trials, respectively. After computing $z$ score by stimulus, those were averaged to yield a single $z$ score per unit per treatment.

Adaptation rates were calculated using trials $6-25$, which is the approximate-linear phase of the adaptation profile in NCM (Phan et al., 2006). For each stimulus, the stimulus firing rate across trials was normalized by the firing on trial 6 (set to 100\%). Then, a linear regression was calculated between trials 6 and 25. For each treatment, the minimum (steepest) adaptation slope across stimuli was used for each unit.

Latency to respond to stimuli were calculated as in Ono et al. (2016). Briefly, for each stimulus, 5-ms PSTH were generated and convolved with a five-point box-filter. The latency to respond to a stimulus was the time after stimulus onset in which the filtered PSTH rose above three standard deviations of the average preceding spontaneous firing period (100 ms). If threshold was not crossed within $400 \mathrm{~ms}$, that stimulus was excluded from analyses.

\section{Experimental design and statistical analysis}

Sample sizes and other variables (sex, hemisphere) are reported above for each experiment's section.

All statistical analyses and plotting were performed using libraries for R and Python, respectively. Summary statistics are shown in mean \pm SEM, unless specified.

Our general statistical approach was to perform GLM (using glmmTMB, DHARMa, and car R packages) followed by ANOVA. Data were initially fitted with Gaussian distributions. Normality of residuals was assessed using DHARMa and Q-Q plot inspection following the GLM fits. If residuals violated normality, data were refit with other distributions (Poisson or negative binomial) and residuals were reassessed based on new distributions. If normality was still violated, data were logtransformed when possible (non-negative, non-zero data) or rankittransformed (Bliss et al., 1956) and the fitting process above was repeated. If residuals were distributed according to GLM distributions, the model was analyzed by ANOVA using Wald $\chi^{2}$ tests. If data still violated residual diagnostics, non-parametric one-way analyses were performed (e.g., Kruskal-Wallis followed by Dunn's post hoc tests, Friedman test, or Wilcoxon signed-rank tests).

For immunofluorescence data, quantifications of the two sections belonging to the same hemisphere and animal were averaged. Response variables were always percentages of the total number of cells (DAPI) or neurons (NeuN). Data were analyzed by GLM followed by ANOVA, with hemisphere and area (dorsal vs ventral NCM) as interacting fixed factors, controlled for area nested within hemisphere nested within subject. In the GABA-D1R-PV analyses, tissue from the right hemisphere of one female was excluded because NCM could not be confidently localized (off-plane section). In the EGR1-D1R-ARO analyses, data from one male in the paired condition could not be quantified because of bad tissue quality after processing. Although animals of both sexes were used, we did not have power to detect sex differences. Nevertheless, no qualitative sex differences were observed. For EGR1 induction analyses, GLM followed by ANOVA was used, with hemisphere, area, and condition (song vs silence; paired vs unpaired) as interacting fixed factors, controlled for area nested within hemisphere nested within subject. To test coexpression proportions, $\chi^{2}$ tests were performed on the total sum of cells across subjects, hemispheres and areas to form a $2 \times 2$ contingency table (e.g., ARO+/ARO- vs D1R+/D1R-). Pearson's $z$ scored residuals were analyzed to obtain corresponding one-tailed $p$ values.

For behavioral analyses, data were grouped by stimulus type (audio or video) and averaged across all 30 trials. Then, we ran GLM followed by ANOVA, with condition (paired vs unpaired) as fixed factor, controlled by subject.

To evaluate whether behaviors predicted EGR1 expression we fit GLMs including hemisphere, area, and condition (paired vs unpaired) as interacting fixed factors and added the behavioral measurement (e.g., screen-time) as an interacting covariate, controlled for area nested within hemisphere nested within subject. To test the effects of behavior on EGR1 expression with increased statistical power, for every combination of the hemisphere $\times$ area interaction we ran individual GLM/ ANOVA with condition and behavioral measurement as interacting factor and covariate.

For patch clamp recordings, minute-by-minute-binned data were analyzed by GLM followed by ANOVA, using time bins (60 s) as a fixed factor controlled over cell nested within subject, and Treatment or Dose as independent fixed factor. Bin 1 corresponded to the minute before SKF treatment; bins 2-8 corresponded to the SKF treatment. Washout was excluded from these analyses and is presented for visualization purposes in all figures. Post hoc Dunnett's tests were used to compare treatment bins with the predrug bin (control).

For in vivo recordings, cell types were classified using 2-D hierarchical clustering (stats $\mathrm{R}$ package) on peak-to-peak duration versus ratio measurements. The optimal number of clusters was determined using the package Nbclust (Charrad et al., 2014) with the gap-statistic method 
(Tibshirani et al., 2001). This established technique contrasts the sum of within-cluster distances with distances from clustering a random uniform distribution. The smallest number of clusters that maximizes the distance (gap) between original and random distributions is selected. This method has been used to separate neural waveforms (Nguyen et al., 2015).

After clustering, each unit's auditory responsiveness was tested by Wilcoxon tests $(30 \times$ spontaneous vs $30 \times$ stimulus trials per song during aCSF infusion). Cells responsive to at least one song were included in all following analyses. Before-drug (PRE) differences among cell types were tested using GLM/ANOVA (controlled over subject) or Kruskal-Wallis tests and Dunn's post hoc tests with Benjamini-Yekutieli false-discovery rate adjustments. Treatment data were analyzed by GLM followed by ANOVA using treatment and cell type as interacting fixed factors, controlled for Unit nested within subject; Tukey's post hoc tests were used when ANOVA effects with more than two factors were significant. To appropriately fit negative-binomial GLMs, firing rate data were summed (instead of averaged) across the 30 stimulus presentations. For a similar reason, to fit Gaussian GLMs, $z$ score data were offset to not contain zero values $[z$ scores $+2 \times \min (z$ scores $)]$ and $\log$-transformed. For simplicity, average firing rates and untransformed $z$ scores are plotted.

Because of statistical power limitations, we performed separate analyses excluding cell type and including hemisphere and sex as factors. However, no systematic sex or hemisphere differences with treatment were detected. Washout (POST) was excluded from all analyses.

\section{Results}

\section{ARO and D1-receptor proteins are coexpressed by NCM neurons}

NCM is a key region for auditory processing in songbirds (Fig. $1 A)$. Previously, we showed that neuroestradiol production in NCM participated in reinforcement-driven auditory association learning (Macedo-Lima and Remage-Healey, 2020). Thus, we hypothesized that reinforcement signaling by the dopaminergic system involved interactions with ARO neurons in NCM. D1R mRNA has been previously reported in NCM (Kubikova et al., 2010), but not its end product, D1R protein. Here, we identified a working antibody for the specific detection of $\mathrm{D} 1 \mathrm{R}$ protein in songbirds as confirmed by the absence of staining when tissue was preincubated with the D1R antigen (Fig. 1B). We found that $\mathrm{D} 1 \mathrm{R}+$ neurons were often found coexpressing ARO, representing $29.6 \%$ and $35.4 \%$ of the ARO + neuronal subpopulation in dNCM and vNCM, respectively (Fig. $1 C$ ). The reciprocal (\% of $\mathrm{D} 1 \mathrm{R}+$ neurons expressing $\mathrm{ARO}$ ) was $26.2 \%$ and $32.3 \%$ in $\mathrm{dNCM}$ and vNCM, respectively. Moreover, the population of $\mathrm{D} 1 \mathrm{R}+/ \mathrm{ARO}+$ neurons represented 6.6 and $10 \%$ of the neuronal population (DAPI nuclei) in $\mathrm{dNCM}$ and $\mathrm{vNCM}$, respectively. A $\chi^{2}$ test analyzing these proportions yielded a significant relationship between D1R and ARO counts $\left(\chi^{2}(1)=10.926, p<0.001\right)$, with double-labeled cells occurring significantly more frequently than expected $(\mathrm{ARO}+/ \mathrm{D} 1 \mathrm{R}+$ Pearson's residual $=2.476, p=$ $0.007)$. Interestingly, we frequently observed $\mathrm{TH}+$ fibers enveloping D1R+/ARO + neurons (for representative images, see Fig. $1 D)$. These data show that D1R protein is prevalent in NCM neurons and suggest that $\mathrm{D} 1 \mathrm{R}+$ neurons represent a significant population in NCM. Moreover, D1R+ neurons are significantly more likely to express ARO+ than D1R- neurons, suggesting an association between estrogen production and dopaminergic signaling.

\section{Song induction of EGR1 in D1R+ and ARO+ neurons}

Next, we examined whether D1R+ and ARO+ NCM neurons were involved in processing of conspecific songs, using the neuroplasticity marker EGR1. Conspecific song playback to birds significantly increased overall EGR1 expression in NCM when compared with birds that experienced silence, which is well established in the literature (Mello et al., 1992; Mello and Clayton, 1994). GLM/ANOVA showed that the degree of song induction of neuronal EGR1 (\% of NeuN) depended on the area and hemisphere (hemisphere $\times$ area $\times$ condition: $\chi^{2}(1)=7.748$, $p=0.005)$. Song exposure increased EGR1 expression in LvNCM (Tukey's post hoc test: song-silence: $21.6 \pm 4.1 \%$ to $8.5 \pm 3.5 \%$ mean $\left.\pm \mathrm{SEM}, t_{(18)}=2.887, p=0.010\right)$ and $\mathrm{R}-\mathrm{dNCM}$ (Tukey's post hoc test: song-silence: $53.0 \pm 6.3 \%$ to $28.1 \pm 6.7 \%$ mean \pm SEM, $\left.t_{(18)}=5.012, p<0.001\right)$. There was also a trend for an increase in L-dNCM, but not R-vNCM (Tukey's post hoc test: song-silence: L-dNCM: $44.8 \pm 11.4 \%$ to $28.5 \pm 2.0 \%$ mean \pm SEM, $t_{(18)}=1.820, p=0.085$, R-vNCM: $18.0 \pm 3.3 \%$ to $12.2 \pm$ $11.4 \%$ mean \pm SEM, $\left.t_{(18)}=1.180, p=0.253\right)$.

We next asked whether EGR1 song induction also occurred in $\mathrm{D} 1 \mathrm{R}+$ and $\mathrm{ARO}+$ neurons. We tested this in three separate GLMs: one for each marker and one for the colabeling. In D1R+ neurons, song exposure increased EGR1 expression when compared with Silence [area $(\mathrm{dNCM}>\mathrm{vNCM}): \chi^{2}(1)=10.202$, $p=0.001$; condition: $\chi^{2}(1)=6.774, p=0.009 ; p>0.08$ in all other main effects; Fig. $2 B$ ]. The same was true for ARO+ and doublelabeled $\mathrm{D} 1 \mathrm{R}+/ \mathrm{ARO}+$ neurons $[\mathrm{ARO}+$ : area $(\mathrm{dNCM}>\mathrm{vNCM})$ : $\chi^{2}(1)=56.723, p<0.001$; condition: $\chi^{2}(1)=13.824, p<0.001$; $p>0.061$ in all other main effects; D1R+/ARO+: area (dNCM $>$ vNCM $): \chi^{2}(1)=3.978, p=0.046$; condition: $\chi^{2}(1)=4.491$, $p=0.034$; area $\times$ condition: $\chi^{2}(1)=3.613, p=0.057$; all other $p>0.190$; Fig. $2 B]$. These data show that song stimulation increased EGR1 expression in $\mathrm{D} 1 \mathrm{R}+, \mathrm{ARO}+$ and double-labeled $\mathrm{D} 1 \mathrm{R}+/ \mathrm{ARO}+$ neurons. These results are consistent with the notion that dopamine and neuroestrogen signaling are each involved in auditory responses to song and may interact in that capacity.

\section{Classical conditioning increases EGR1 expression specifically in $\mathrm{D} 1 \mathrm{R}+$ neurons}

We were interested in whether our novel classical conditioning paradigm would induce expression of EGR1 in NCM neurons, so we compared expression levels between the paired group (sound followed by video) and the unpaired group (sound and video presented at random; Fig. $2 C, D$ ). We found that overall EGR1 neuronal induction (i.e., total EGR1 as a \% of NeuN) was not affected by condition [area $(\mathrm{dNCM}>\mathrm{vNCM}): \chi^{2}(1)=$ 24.726, $p<0.001$; condition: $\chi^{2}(1)=2.154, p=0.142 ; p>0.187$ in all other main effects; data not shown]. However, the frequency of double-labeled D1R+/EGR1+ neurons was significantly increased by condition (condition: $\chi^{2}(1)=6.370$, $p=0.012 ; p>0.150$ in all other main effects; Fig. $2 E$ ). This effect was not observed with $\mathrm{ARO}+/ \mathrm{EGR} 1+$ neurons [area $(\mathrm{dNCM}>\mathrm{vNCM}): \chi^{2}(1)=22.430, p<0.001$; condition: $\chi^{2}(1)=$ 1.027, $p=0.311 ; p>0.290$ in all other main effects] or with $\mathrm{D} 1 \mathrm{R}+/ \mathrm{ARO}+/ \mathrm{EGR} 1+$ neurons [condition: $\chi^{2}(1)=1.593$, $p=0.207 ; p>0.232$ in all other main effects].

We noticed that D1R+/EGR1+ expression levels for both unpaired and paired groups seemed qualitatively lower than the song playback reference control. However, a GLM including all reference and experimental groups only found differences between unpaired and song (condition: $\chi^{2}(1)=20.957$, $p<0.001$; area: $\chi^{2}(1)=5.777, p=0.016 ; p>0.173$ for all other main effects; Tukey's post hoc test for condition: unpaired-song: $t_{(54)}=-4.484, p<0.001$; silence-song: $t_{(54)}=-2.790, p<0.0035$; paired-unpaired: $t_{(54)}=2.481, p=0.074$; all other $p>0.174$; Fig. $2 E)$. This suggests that classical conditioning increased EGR1 
expression in D1R + to statistically similar levels to song exposure, while silence and unpaired treatments remained low.

We note that we observed a significant interaction between hemisphere and condition on our NeuN counts [area (dNCM $>$ vNCM): $\chi^{2}(1)=9.910, p=0.002$; hemisphere $\times$ condition: $\chi^{2}(1)=6.376, p=0.012 ; p>0.068$ in all other main effects. Tukey's post hoc test paired vs unpaired: L hemisphere: $t_{(33)}=$ $-2.217, p=0.034$, R hemisphere: $\left.t_{(33)}=0.226, p=0.822\right]$. We ran a GLM/ANOVA including NeuN counts as a covariate to examine its impact on D1R+/EGR1+ frequency. We found that NeuN numbers did not account for the differences we observed above, i.e., that classical conditioning increased the frequency of $\mathrm{D} 1 \mathrm{R}+/ \mathrm{EGR} 1+$ expression in NCM neurons $\left(\mathrm{NeuN}: \chi^{2}(1)=\right.$ $0.998, p=0.318$; condition: $\chi^{2}(1)=6.748, p=0.009 ; p>0.229$ in all other main effects). We are unable to explain this interaction between condition and hemisphere in NeuN expression in our sample. Still, this result has little bearing on our main conclusion that classical conditioning increases EGR1 expression in D1R+ neurons.

Next, we wanted to explore whether the increase in EGR1 expression in $\mathrm{D} 1 \mathrm{R}+$ neurons in our paired group could be explained by any behavioral parameter. Specifically, we were concerned that differences in "reward" exposure (i.e., more time looking at the screen) could underlie the difference we observed between treatment groups. Indeed, we individually tested all behaviors we scored and found that average screen-time (beak directions toward left, right or screen; see Materials and Methods) was significantly higher in the paired group (GLM/ ANOVA: $\chi^{2}(1)=4.820, p=0.028$; Fig. $2 F$ ). Similarly, state behaviors that reflected distractibility toward the video (eyes closed, eating, drinking, continuously flying, beak direction toward camera) were higher in the unpaired group (GLM/ ANOVA: $\left.\chi^{2}(1)=10.08, p=0.001\right)$.

To formally test whether screen-time predicted EGR1 expression in D1R+ neurons, we performed a GLM including screentime as a covariate. Indeed, screen-time was a predictor of $\mathrm{D} 1 \mathrm{R}+/ \mathrm{EGR} 1+$ expression, but there was a significant interaction between all factors (screen-time: $\chi^{2}(1)=9.816, p=0.003$; hemisphere $\times$ area $\times$ condition $\times$ screen-time: $\chi^{2}(1)=4.137$, $p=0.042$; all other $p>0.068$; Fig. $2 F)$. Distractibility did not predict D1R+/EGR1+ (distractibility: $\chi^{2}(1)=2.441, p=0.118$; all other $p>0.153)$.

Because we observed a four-way interaction with hemisphere $\times$ area $\times$ condition $\times$ screen-time, we retested these data using GLMs for each hemisphere $\times$ area combination. We found that condition (paired vs unpaired) and not screentime was a significant predictor of D1R+/EGR1+ neurons in the L-vNCM (condition: $\chi^{2}(1)=4.765, p=0.029$; screen-time: $\chi^{2}(1)=2.280, p=0.131$; condition $\times$ screen-time: $\chi^{2}(1)=0.903$, $p=0.342$ ), indicating that associating sounds with reinforcement drove EGR1 in D1R-expressing neurons. Conversely, in the LdNCM, screen-time (but not condition) was a significant predictor of EGR1 in D1R + neurons (condition: $\chi^{2}(1)=0.008, p=0.929$; screen-time: $\chi^{2}(1)=3.926, p=0.047$; condition $\times$ screen-time: $\left.\chi^{2}(1)=1.884, p=0.170\right)$. In the right hemisphere, neither factor nor interactions were statistically significant (all $p>0.231$ ). ANOVA results are illustrated in Figure $2 G$.

We also asked whether EGR1 expression was being driven by classical conditioning in only a subset of D1R + with respect to ARO presence. For D1R+/ARO- neurons we found an effect of classical conditioning (condition: $\chi^{2}(1)=6.8724, p=0.009$; paired $>$ unpaired). However, when controlling by screen-time, we found that this effect was driven by screen-time and not by condition (hemisphere $\times$ screen-time: $\chi^{2}(1)=6.542, p=0.011$; hemisphere $\times$ area: $\chi^{2}(1)=4.867, p=0.027$; all other $p>0.11$ ). In D1R+/ARO + cells EGR1 expression was not affected by classical conditioning as stated above, but screen-time did affect its distribution (screen-time: $\chi^{2}(1)=7.492, p=0.006$; area $\times$ hemisphere $\times$ condition $\times$ screen-time: $\chi^{2}(1)=4.447, p=0.035$; all other $p>0.190)$. However, 2 -factor GLMs controlling by hemisphere and area only yielded a trend for screen-time in the R$\mathrm{dNCM}(p=0.056)$ and R-vNCM $(p=0.097)$ and no other effects (all other $p>0.139$ ). Therefore, the effects of classical conditioning we observed can be explained by the increase of EGR1 expression in $\mathrm{D} 1 \mathrm{R}+$ neurons that can be either ARO+ or ARO(both types contributing to the effect).

In summary, screen-time was a strong predictor of EGR1 expression in $\mathrm{D} 1 \mathrm{R}+$ neurons (both $\mathrm{ARO}+$ and $\mathrm{ARO}-$ ), but this effect was particular to the L-dNCM. Importantly, classical conditioning increased EGR1 expression in D1R+ neurons in the LvNCM which suggests that association during classical conditioning is hemisphere and subregion specific. This nuanced regional modulation might have emerged in part because of the nature of our auditory stimulus (2-kHz tone) and the tonotopic organization of NCM (Müller and Leppelsack, 1985).

\section{$\mathrm{D} 1 \mathrm{R}+$ neurons are predominantly GABA+}

Next, we wanted to characterize the neurotransmitter profile of $\mathrm{D} 1 \mathrm{R}+$ cells in NCM. We found that the majority $(58.7 \%$ and $64.2 \%$ ) of $\mathrm{D} 1 \mathrm{R}+$ neurons are GABA + , and these colabeled neurons represent $21.9 \%$ and $26.4 \%$ of the neuronal population in $\mathrm{dNCM}$ and vNCM, respectively. The reciprocal was also true, such that $54.6 \%(\mathrm{dNCM})$ and $56.5 \%(\mathrm{vNCM})$ of GABA+ neurons contained D1R (Fig. 3A). A $\chi^{2}$ test analyzing these proportions showed that $\mathrm{D} 1 \mathrm{R}+/ \mathrm{GABA}+$ co-expression was significantly more frequent than expected $\left(\chi^{2}(1)=319.660, p<0.001\right.$; Pearson's residual $=$ 9.775, $p<0.001)$. Similarly, we observed that the majority $(\sim 55 \%)$ of PV+ neurons also express D1R, combining vNCM and dNCM (Fig. $3 B$ ), and that this neurochemical phenotype represented $\sim 4 \%$ of the entire NCM neuronal population (Fig. 3A). The D1R+/PV+ cell population proportion is also more frequent than expected $\left(\chi^{2}(1)=22.288, p<0.001 ;\right.$ Pearson's residual $=3.470, p<$ $0.001)$. These results show that $\mathrm{D} 1 \mathrm{R}+$ neurons are predominantly GABA + and represent a significant subpopulation in NCM. Of note, our data show that the majority of PV + neurons express D1Rs, which suggests this subpopulation is of particular interest for dopamine modulation of auditory processing.

\section{D1R activation reduces the amplitude of GABAergic currents in NCM in vitro}

The anatomic findings above led to the hypothesis that activating D1Rs would modulate GABAergic neurotransmission. Thus, we recorded spontaneous PSCs from neurons in NCM in vitro (Fig. $4 A)$.

Inhibitory currents were isolated with the AMPA receptor antagonist DNQX (sIPSCs) and were confirmed to be GABAergic by GABA receptor antagonist bicuculline application at the end of recordings. In separate sets of cells, we either applied $10 \mu \mathrm{M}$ SKF or nothing (rundown) to the bath. Only recordings with low and stable series resistance throughout the baseline and drug/ rundown period (Rs $<40 \mathrm{M} \Omega ;<20 \%$ change; see Materials and Methods) were included (Rs $=16.971 \pm 0.826 \mathrm{M} \Omega$, mean \pm SEM of all recording periods). We obtained stable recordings of 11 cells (six animals), seven of which (from five animals) received the SKF treatment. 

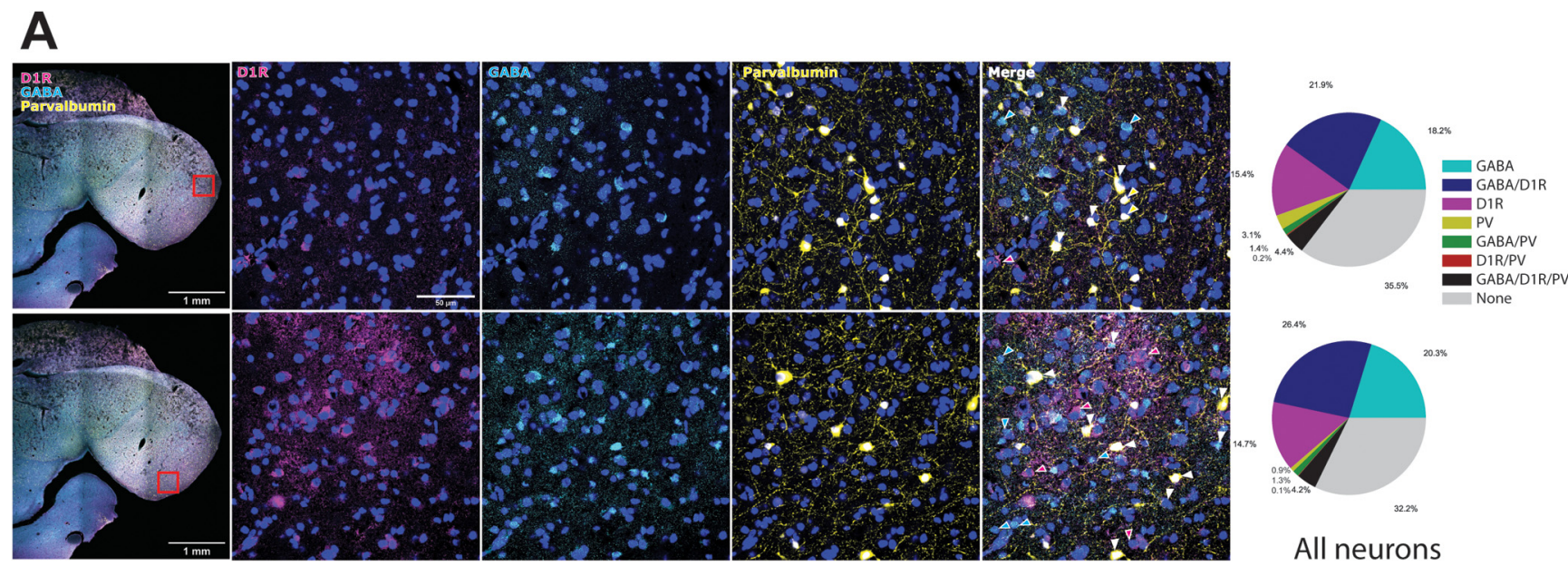

All neurons

B
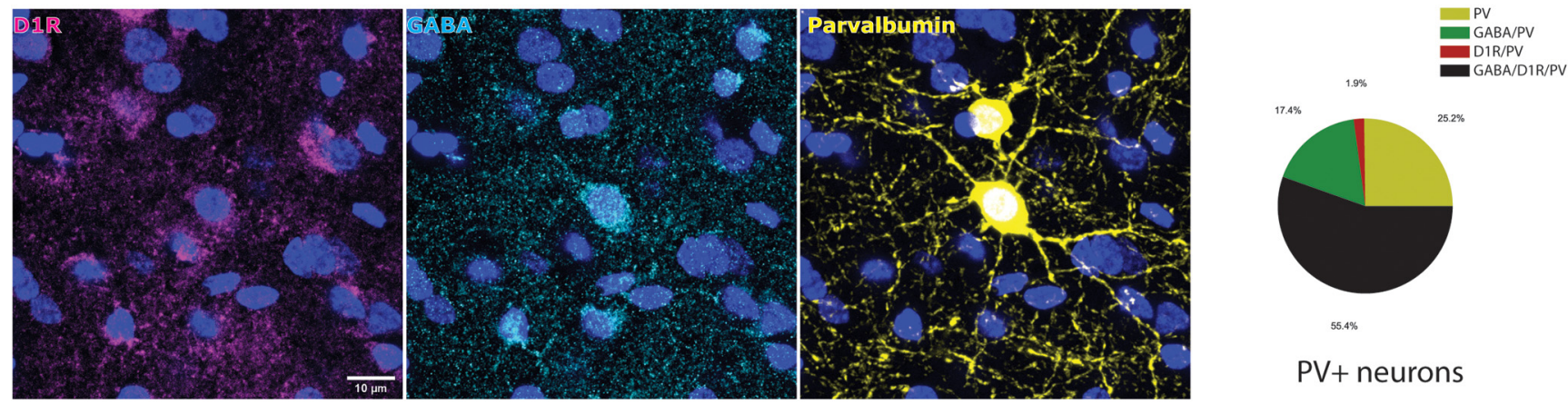

$\mathrm{PV}+$ neurons

Figure 3. Dopamine D1R-containing neurons are predominantly GABA+. $A$, Triple immunofluorescence stain for D1R, GABA, and PV. Magenta, cyan, and yellow arrows indicate single-labeled D1R, GABA, and PV+ neurons, respectively. White arrows indicate triple labeling. $\boldsymbol{B}$, The majority of PV + neurons also express GABA and D1Rs.

For amplitude measurements, GLM/ANOVA analyses comparing treatments (SKF or rundown) detected a significant interaction between Time and Treatment (time: $\chi^{2}(7)=27.781$, $p<0.001$; treatment: $\chi^{2}(1)=0.085, p=0.770$; time $\times$ treatment: $\chi^{2}(7)=15.592, p=0.029$; Fig. $4 B$ ). Dunnett's post hoc tests revealed a significant reduction in sIPSC amplitude only in SKF treated neurons (vs before-treatment: $p<0.05$ between minutes 4-7 of SKF). For frequency measurements, GLM/ANOVA analyses did not detect significant differences between SKF-38393 and rundown, but detected a significant reduction of sIPSC frequency over time (time: $\chi^{2}(7)=27.454, p<0.001$; treatment: $\chi^{2}(1)=0.096, p=0.757$; time $\times$ treatment: $\chi^{2}(7)=1.735$, $p=0.973$; Dunnett's post hoc test vs before-treatment: $p<0.05$ between minutes 5 and 7; Fig. $4 B$ ). These results show that SKF38393 treatment significantly reduced the amplitude of GABAergic currents. The reduction in frequency observed during SKF-38393 treatments did not differ from rundowns indicating that, regardless of treatment, the number of detected sIPSCs decayed over time.

We were unable to test a lateralization effect for this dataset, because after exclusion of unstable recordings, our sample consisted of two cells from the left and five from the right hemisphere.

\section{D1R activation reduces the amplitude but increases the} frequency of glutamatergic currents in $\mathrm{NCM}$ in vitro Our anatomic findings suggest that $\sim 38 \%$ of D1R-positive neurons are non-GABAergic (i.e., putatively glutamatergic; see Fig. $3 A$ ), which led to the hypothesis that activating D1Rs would also directly modulate glutamatergic currents in NCM. Therefore, we recorded excitatory NMDA/AMPA/kainate currents (sEPSCs) isolated in $0-\mathrm{Mg}^{2+}$ bath containing the $\mathrm{GABA}_{\mathrm{a}}$-receptor antagonist bicuculline. For amplitude measurements, only recordings with low and stable series resistance throughout the baseline and drug periods (Rs $<40 \mathrm{M} \Omega ;<20 \%$ change; see Materials and Methods) were included ( $\mathrm{Rs}=28.770 \pm 2.216 \mathrm{M} \Omega$, mean \pm SEM of all recording periods). For frequency measurements, series resistance change was not employed as quality control because the observed currents were very high amplitude and highly unlikely to go undetected with small changes in Rs. Still, only recordings with $\mathrm{Rs}<40 \mathrm{M} \Omega$ were included (Rs $=26.880 \pm 1.428 \mathrm{M} \Omega$, mean $\pm \mathrm{SEM}$ of all recording periods). We obtained stable recordings from nine cells (four animals) for amplitude and 16 cells (eight animals) for frequency.

For these experiments we used two doses $(10$ and $50 \mu \mathrm{M})$ of SKF-38393 in different sets of cells. In these experiments we performed rundown experiments before SKF-38393 treatment in a subset of cells ( $n=4$ for amplitude; $n=10$ for frequency; see Materials and Methods). Both amplitude and frequency of sEPSCs were stable during $7 \mathrm{~min}$ before treatment (GLM/ ANOVA: amplitude: $\chi^{2}(6)=4.620, p=0.593$; frequency: $\chi^{2}(6)=$ 6.916, $p=0.329$; Fig. $4 C$ ). Currents were confirmed to be NMDA dependent at the end of the recordings.

For amplitude measurements following SKF-38393 treatment, we performed GLM/ANOVA on the effect of different doses of SKF-38393 over time. These analyses showed a reduction in the amplitude of sEPSCs over time because of treatment, and no difference between the two SKF doses (time: $\chi^{2}(7)=48.729$, $p<0.001$; treatment: $\chi^{2}(1)=0.0046, p=0.9461$; time $\times$ treatment: $\chi^{2}(7)=2.950, p=0.890$; Dunnett's post hoc test vs before- 

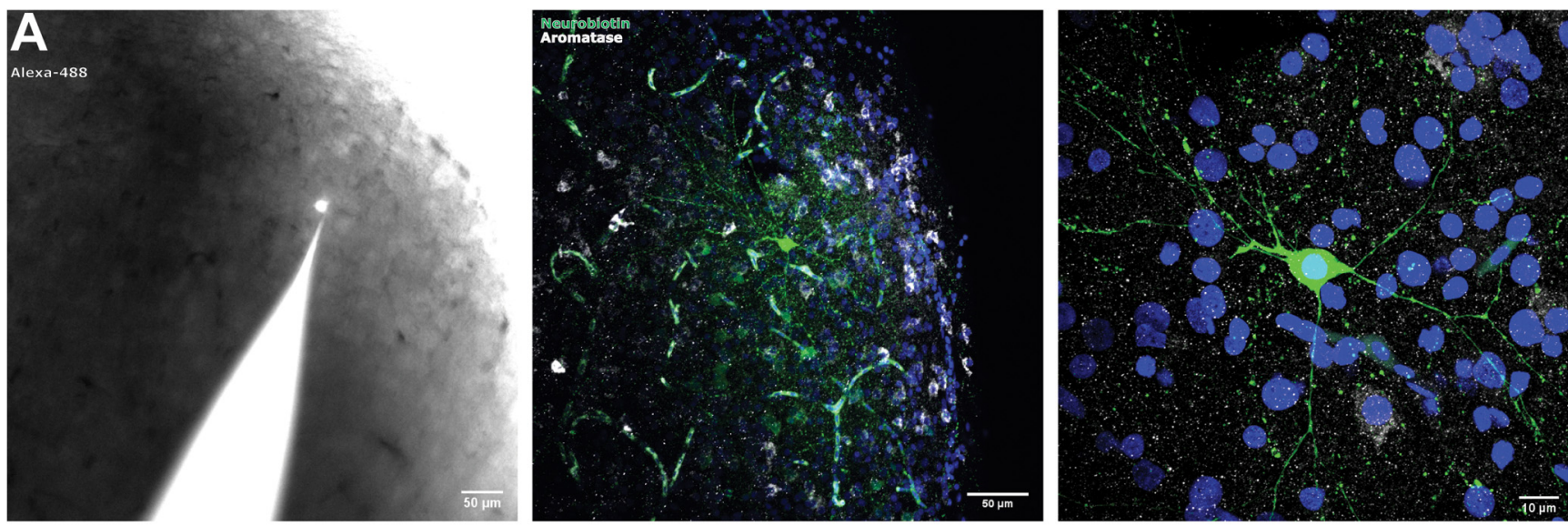

B
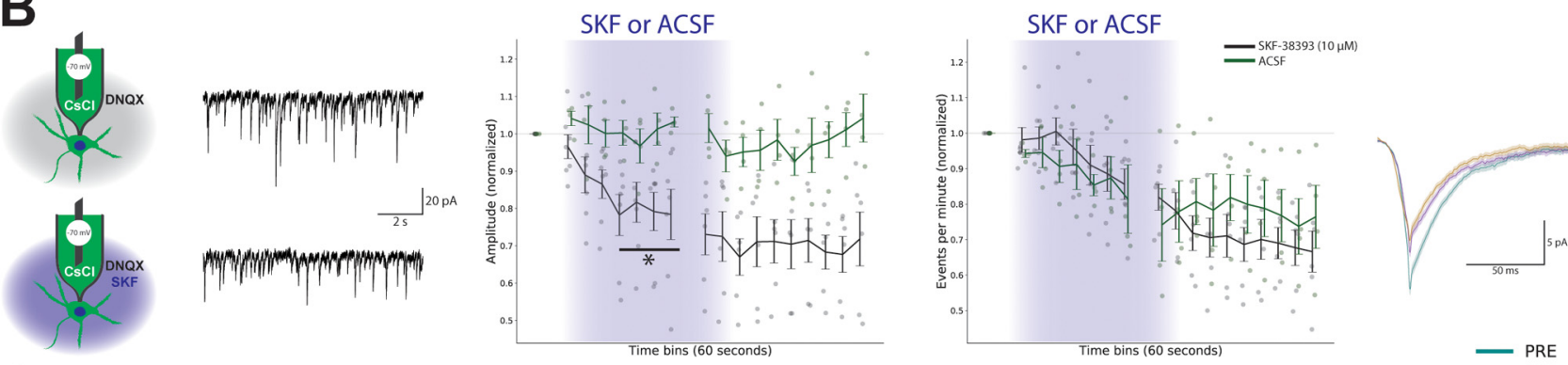

C
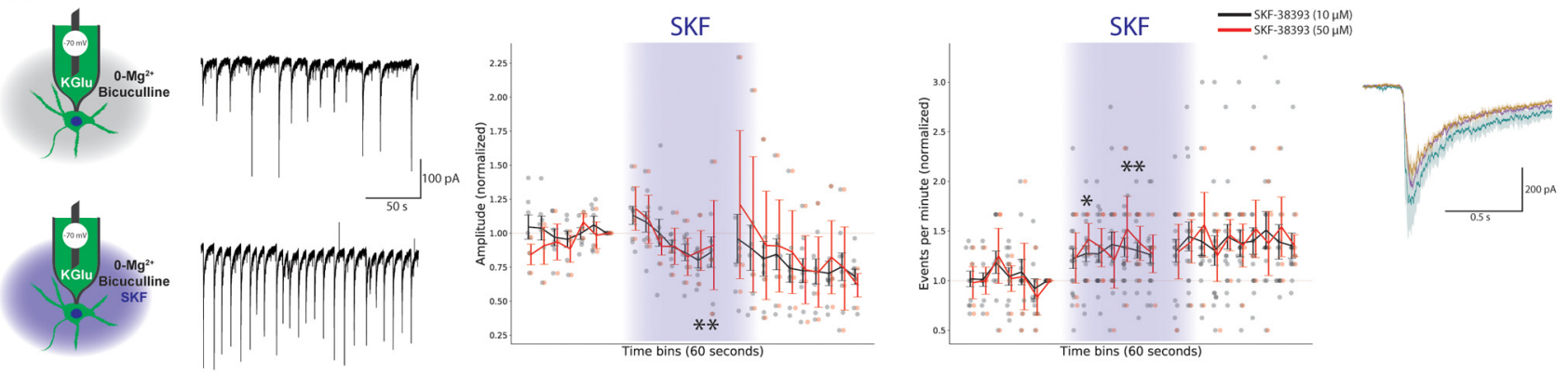

Figure 4. Dopamine D1R activation with SKF-38393 reduces spontaneous GABAergic (SIPSC) and glutamatergic (SEPSC) current amplitudes but increases sEPSC frequency. $\boldsymbol{A}$, Representative neuron in NCM visualized during (left; Alexa Fluor 488) and after recording (neurobiotin-streptavidin 488 stain; AR0 stain to confirm NCM location). $\boldsymbol{B}$, Representative sIPSCS recording from the same NCM neuron, before and during $10 \mu \mathrm{m}$ SKF-38393 infusion (left panel). Amplitude of sIPSCs (middle panel) was significantly different from rundowns (aCSF) and reduced from baseline by SKF (4-7 min). Frequency (right panel) was also reduced but did not differ from rundown experiments (5-7 min). Waveforms show representative sIPSC (mean \pm SEM during 1 min) before (PRE), on the last minute of (SKF) and on the last minute after (POST) drug infusion. C, Representative AMPA/NMDA/kainate sEPSCs recording from the same NCM neuron, before and during $10 \mu \mathrm{m}$ SKF-38393 infusion (left panel). Amplitude of sEPSCs (middle panel) was significantly reduced by 10 and $50 \mu \mathrm{m}$ (no dose difference) SKF (6-7 min). In addition, frequency (right panel) was increased by SKF $(2,5 \mathrm{~min})$. In these experiments, rundowns were performed before treatment and did not significantly change. Waveforms show representative sEPSC (mean \pm SEM during $1 \mathrm{~min}$ ) before (PRE), on the last minute of (SKF), and on the last minute after (POST) drug infusion; ${ }^{*} p<0.05$. Downward current traces in $\boldsymbol{B}, \boldsymbol{C}$ represent depolarizing current deflections.

drug: $p<0.05$ on minutes $6-7$ of SKF; Fig. $4 C)$. For frequency measurements, GLM/ANOVA analyses showed an increase in sEPSC frequency over time, and again no difference between the two SKF doses (time: $\chi^{2}(7)=23.394, p=0.001$; treatment: $\chi^{2}(1)=$ 3.140, $p=0.076$; time $\times$ treatment: $\chi^{2}(7)=4.358, p=0.738$; Dunnett's post hoc test vs before-drug: $p<0.05$ on minutes 2, 5, and 6 of SKF; Fig. 4C).

We also tested whether we could detect a lateralization effect. We recorded from 6 cells from the left (five animals) and 10 cells from the right (eight animals) hemisphere. For amplitude measurements, stable recordings were obtained from four cells from the left (three animals) and five cells from the right (three animals) hemispheres (see Materials and Methods). Hemisphere was included as an interacting factor with Time (and as a random variable nested within subject). We did not find significant effects of hemisphere for either amplitude (time: $\chi^{2}(7)=54.660$, $p<0.001$; hemisphere: $\chi^{2}(1)=0.423, p=0.516$; time $\times$ hemisphere: $\left.\chi^{2}(7)=10.977, p=0.140\right)$ or frequency (time: $\chi^{2}(7)=$ 21.281, $p=0.003$; hemisphere: $\chi^{2}(1)=2.262, p=0.133$; time $\times$ hemisphere: $\left.\chi^{2}(7)=11.943, p=0.102\right)$.

In summary, D1R activation in vitro reduced the amplitude of both GABA and glutamatergic spontaneous currents, while also increasing the frequency of the latter. These findings establish a role for dopamine modulation of network excitation and inhibition in $\mathrm{NCM}$, and predict that D1R activation in vivo causes differential effects depending on cell type (i.e., downregulate vs upregulate GABAergic and glutamatergic neuron firing, respectively).

Cell-type separation based on waveform measurements for in vivo recordings

We isolated 107 single-units from nine adult birds in awake headfixed recordings (Fig. 5) using acute recording 32-channel 
microdrives coupled to retrodialysis probes (RetroDrives; Fig. $6 A, B$; see Materials and Materials and Methods). We measured peak-topeak duration and ratio of each unit and analyzed the data using an unsupervised hierarchical clustering algorithm (see Materials and Methods; Fig. 5A). The gap-statistic results show that the variance in clustering was best explained by four clusters, which we named narrow-spiking 1 and 2, and broad-spiking 1 and 2 (NS1, NS2, BS1, BS2, respectively). Cell types differed significantly in waveform peak-to-peak duration (GLM/ANOVA: $\chi^{2}(3)=299.970, p<0.001$; Tukey's post hoc test: all $p<0.01$ ) and ratio (GLM/ANOVA: $\chi^{2}(3)=294.880, p<0.001$; Tukey's post hoc test: all $p<0.001$ except BS1-BS2 where $p=0.972$ ). In summary, waveform peak-topeak duration followed the pattern $\mathrm{NS} 1<\mathrm{NS} 2<\mathrm{BS} 1<\mathrm{BS} 2$ and waveform peak-to-peak ratio followed $\mathrm{BS} 2<\mathrm{BS} 1=\mathrm{NS} 1<\mathrm{NS} 2$.

The classification commonly used in the literature of narrowspiking and broad-spiking neurons in songbird pallium uses only peak-to-peak duration and a division boundary of $\sim 0.4 \mathrm{~ms}$ (Schneider and Woolley, 2013; Yanagihara and YazakiSugiyama, 2016; Vahaba et al., 2017; Cazala et al., 2019; but see Bottjer et al., 2019). Using both peak-to-peak duration and ratio, we provide evidence of two further subdivisions; therefore, we named our clusters to extend the previous classification: NS1 and NS2, narrow-spiking; and BS1 and BS2, broad-spiking.

Following clustering, 15 non-auditory-responsive cells were excluded from the analyses (see Materials and Methods) and 92 units were further analyzed. This sample consisted of $21(\mathrm{~L})$ and $23(\mathrm{R})$ units from females and $34(\mathrm{~L})$ and $14(\mathrm{R})$ units from males. In this sample, BS2 were more numerous (34.8\%) followed by BS1 (27.2\%), NS1 (26.1\%), and NS2 (12\%; Fig. 5A). Representative PSTHs and adaptation slopes of a NS1 and a BS1 are shown in Figure $5 B$.

The baseline physiological profiles assessed during aCSF infusion showed clear segregation by cell type classification (Fig. $5 C$ ). GLM/ANOVA analyses showed that cell types differed in spontaneous firing rates $\left(\chi^{2}(3)=50.554, p<0.001\right.$; Tukey's post hoc test: $p<0.05$ in NS1-BS1, NS1-BS2, NS2-BS2 and BS1-BS2), stimulus firing rates $\left(\chi^{2}(3)=86.754, p<0.001\right.$; Tukey's post hoc test: $p<0.05$ in NS1-NS2, NS1-BS1, NS1-BS2 and NS2-BS2), $z$ scores $\left(\chi^{2}(3)=36.169, p<0.001\right.$; Tukey's post hoc test: $p<0.05$ in NS1NS2, NS1-BS1 and NS1-BS2), adaptation slopes $\left(\chi^{2}(3)=10.465\right.$, $p=0.015$; Tukey's post hoc test: $p<0.05$ in NS2-BS2), latencies to respond $\left(\chi^{2}(3)=12.166, p=0.006\right.$; Tukey's post hoc test: $p<0.05$ in NS1-BS2), and in the $\%$ of songs responded to $\left(\chi^{2}(3)=22.785\right.$, $p<0.001$; Tukey's post hoc test: $p<0.05$ in NS1-BS1 and NS1BS2). Therefore, the four cell types clustered by waveform shape in our recordings also differed in physiological profile.

Broadly speaking, NS1 cells had highly symmetrical and narrow action potentials, high firing rates and $z$ scores, as well as fast response latencies and lower selectivity, which all parallel features of mammalian cortical high-firing PV + inhibitory interneurons. Compared with NS1, NS2 cells had less narrow and symmetrical waveforms with lower firing rates, which resemble properties of mammalian cortical low-firing somatostatin+ or VIP+ inhibitory interneurons (Tremblay et al., 2016). BS1 and BS2 had broader and less symmetrical waveforms and lower firing rates than NS1/NS2. Additionally, they showed longer latencies to respond to stimuli and higher selectivity, all features that resemble those of mammalian cortical excitatory projection neurons (Atencio and Schreiner, 2008; Hromádka et al., 2008; Wu et al., 2008; Tsunada et al., 2012).

D1R activation reduces spontaneous and stimulus firing of NS1 but increases spontaneous firing of BS1 neurons in vivo Our in vitro experiments generated clear predictions about differential D1R activation on narrow- versus broad-spiking neurons in vivo (see above). We therefore analyzed how SKF38393 (SKF; $0.2 \mathrm{~mm}$ ) affected single-unit responses to sound playback (timeline in Fig. 6). Representative PSTHs for NS1 and BS1 cells are shown in Figure 7A. We observed effects of D1R activation that were selective for cell waveform phenotypes in NCM.

For spontaneous firing rate, GLM/ANOVA analyses comparing Treatment and Cell-type showed that SKF reduced the firing of NS1 and tended to increase the firing of BS1 (treatment: $\chi^{2}(1)=0.011, p=0.916$; cell type: $\chi^{2}(3)=54.694, p<0.001$; treatment $\times$ cell type: $\chi^{2}(3)=12.866, p=0.005$; Tukey's post hoc test: PRE-SKF: NS1: $t_{(173)}=2.702, p=0.007$; NS2: $t_{(173)}=0.712$, $p=0.477$; BS1: $t_{(173)}=-1.758, p=0.079 ; \mathrm{BS} 2: t_{(173)}=-1.155$, $p=0.248$; Fig. $7 B$ ). Because of the trend we observed, to assess whether a treatment effect could be detected in BS1 units with increased statistical power, we performed a single-factor GLM/ ANOVA only on BS1 units, which yielded a significant increase in spontaneous firing because of $\operatorname{SKF}\left(\chi^{2}(1)=6.008, p=0.014\right)$. Singlefactor GLMs for NS2 and BS2 yield non-significant treatment effects $\left(\mathrm{NS} 2: \chi^{2}(1)=0.001, p=0.972\right.$; BS2: $\left.\chi 2(1)=2.650, p=0.104\right)$.

For stimulus firing rate, SKF decreased firing of NS1 cells (treatment: $\chi^{2}(1)=0.734, p=0.392$; cell type: $\chi^{2}(3)=53.731$, $p<0.001$; treatment $\times$ cell type: $\chi^{2}(3)=9.043, p=0.029$; Tukey's post hoc test: PRE-SKF: NS1: $t_{(173)}=2.753, p=0.007$; NS2: $t_{(173)}=0.783, p=0.435$; BS1: $t_{(173)}=-1.232, p=0.220$; BS2: $t_{(173)}=-0.183, p=0.855$; Fig. $\left.7 C\right)$.

To explore further the change in spontaneous and stimulus firing because of SKF, we plotted a correlation between the $\%$-change in spontaneous versus stimulus firing induced by SKF (Fig. 7D). Values above 0 in either axis indicate an increase in firing because of SKF. Note that on average BS1 and BS2 data points were situated above 0 in both axes, whereas NS1 and NS2 were below 0 . Changes in spontaneous versus stimulus firing were also highly correlated (Pearson's $r=0.876, p<0.001$ ). Interestingly, the regression line slope's $95 \%$ confidence interval $[0.693,0.875]$ falls lower than and does not include the slope of the identity line (slope $=1$ ), which suggests that spontaneous firing was more affected by SKF than stimulus firing.

For stimulus response $z$ scores, GLM/ANOVA analyses showed that SKF decreased overall $z$ scores regardless of cell type (treatment: $\chi^{2}(3)=8.657, p=0.003$; cell type: $\chi^{2}(3)=46.855, p<0.001$; treatment $\times$ cell type: $\chi^{2}(3)=4.443, p=0.217$; Fig. $\left.7 E\right)$.

Finally, SKF-38393 treatment increased overall latency to respond (treatment: $\chi^{2}(3)=5.754, p=0.016$; cell type: $\chi^{2}(3)=$ 13.597, $p=0.004$; treatment $\times$ cell type: $\chi^{2}(3)=2.248, p=0.523$; Fig. $7 F$ ) and decreased the $\%$ of songs units responded to (treatment: $\chi^{2}(3)=8.929, p=0.003$; cell type: $\chi^{2}(3)=31.935$, $p<0.001$; treatment $\times$ cell type: $\chi^{2}(3)=4.409, p=0.221$; Fig. $7 G)$ regardless of cell type.

Altogether, these results show that the D1R agonist SKF38393 affects the response properties of NCM cell types differentially. Notably, as predicted by our in vitro experiments, D1R activation reduces the firing of putative inhibitory neurons (NS1) while increasing the firing of putative excitatory neurons (BS1). Furthermore, in all cell types D1R activation decreased $z$ scores, increased latency to respond and decreased the \% of songs units responded to, which might represent direct or indirect consequences of the shift in inhibitory/excitatory balance induced by tonic D1R activation.

\section{D1R activation sharply reduces neuronal SSA in vivo}

NCM neurons show SSA when birds are presented repetitions of the same stimuli, which are thought to reflect short/medium- 


\section{A}
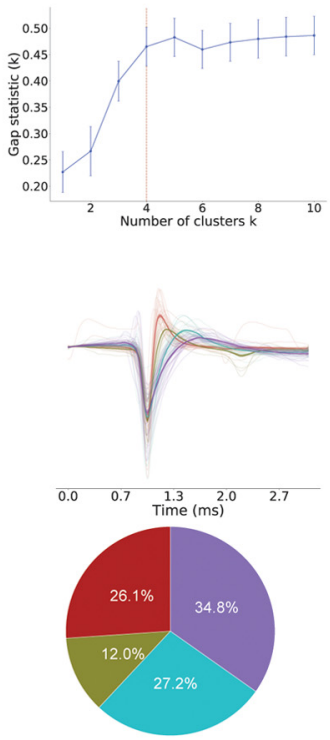

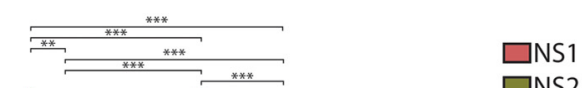

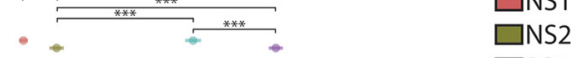

$\square \mathrm{BS} 1$

$\square$ BS2

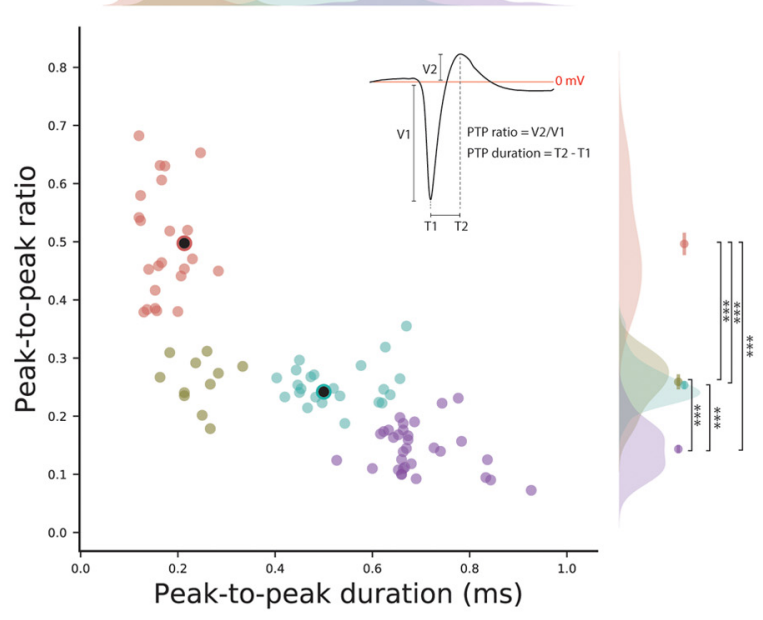

B
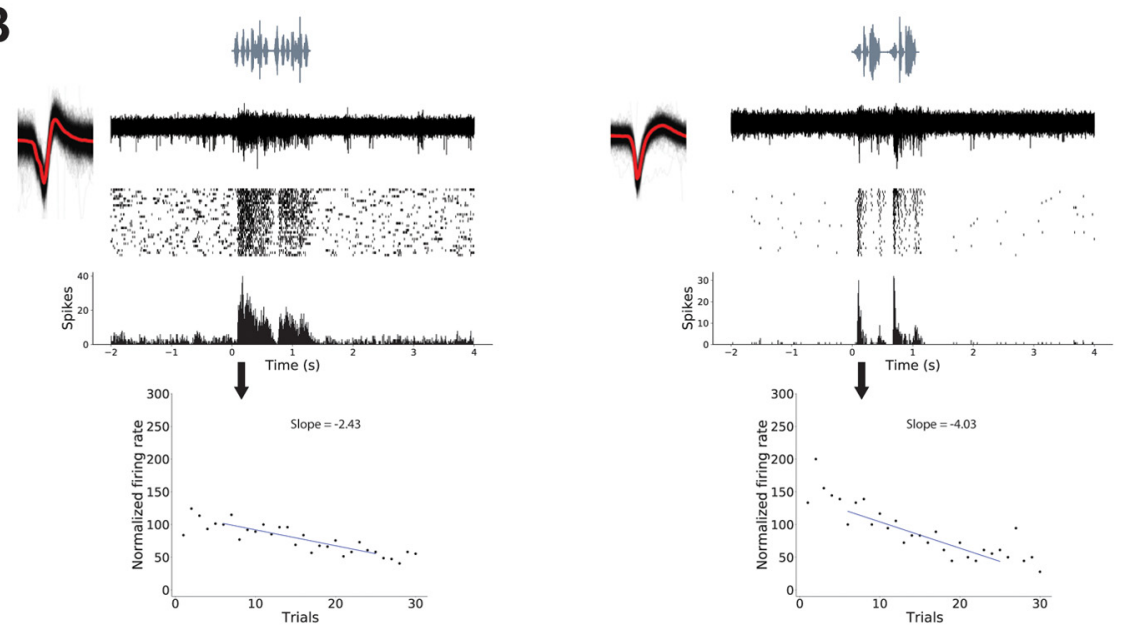

C
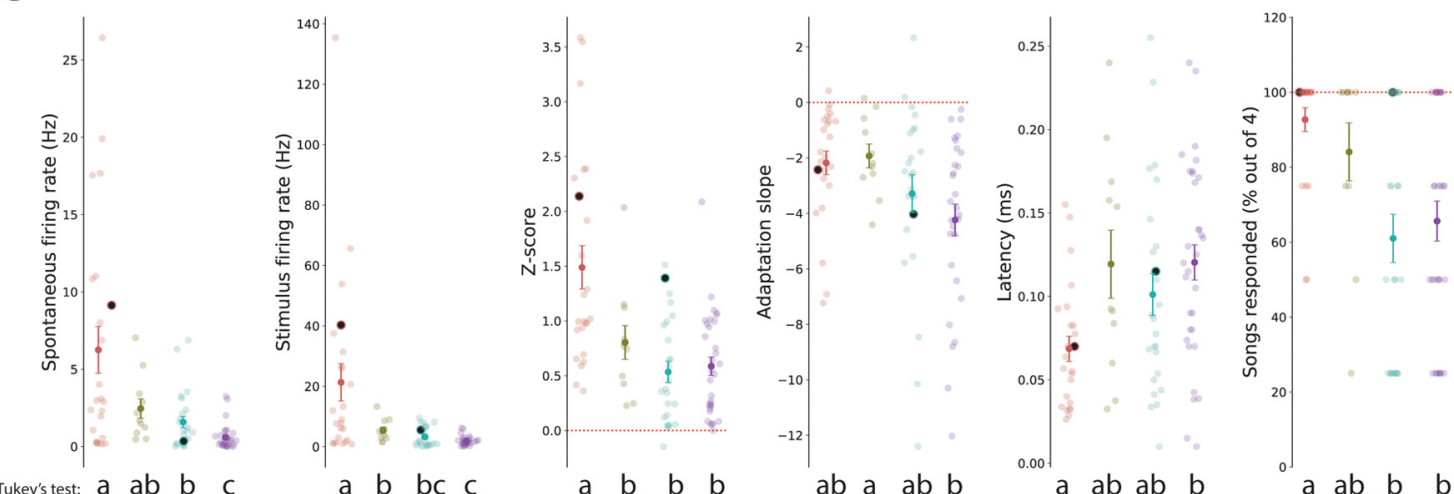

Figure 5. Cell type separation based on waveform measurements in in vivo recordings. $\boldsymbol{A}$, Unsupervised hierarchical clustering results (gap statistic) using peak-to-peak ratio versus duration (top left); separation into four clusters best explained the variance in the data. Traces illustrate all waveforms in our sample by cell type (middle left). Shaded lines are the average waveforms of each unit in our sample; opaque lines and shading are the grand average \pm SEM of those. The pie chart represents the proportions of units within each cluster in our sample (bottom left). The correlation plot shows the four distinct clusters and the kernel-density estimations of the distributions along single-axis with mean \pm SEM on top. $\boldsymbol{B}$, Representative PSTHs and adaptation slopes of NS1 (left) and BS1 (right) cell types. Representative examples in B are highlighted in all graphs according to cluster type. C, Physiology parameters of the different cluster types. From left to right, Spontaneous and stimulus firing rate, $z$ score, adaptation slope, latency to respond to song, and percentage of songs responded to. Tukey's post hoc test results are shown at the bottom. Significant differences $(p<0.05)$ are assigned different letters; ${ }^{*} p<0.05,{ }^{* *} p<0.01,{ }^{* * *} p<0.001$. Highlighted datapoints in $\boldsymbol{A}, \boldsymbol{C}$ depict the two representative cells in $\boldsymbol{B}$. 

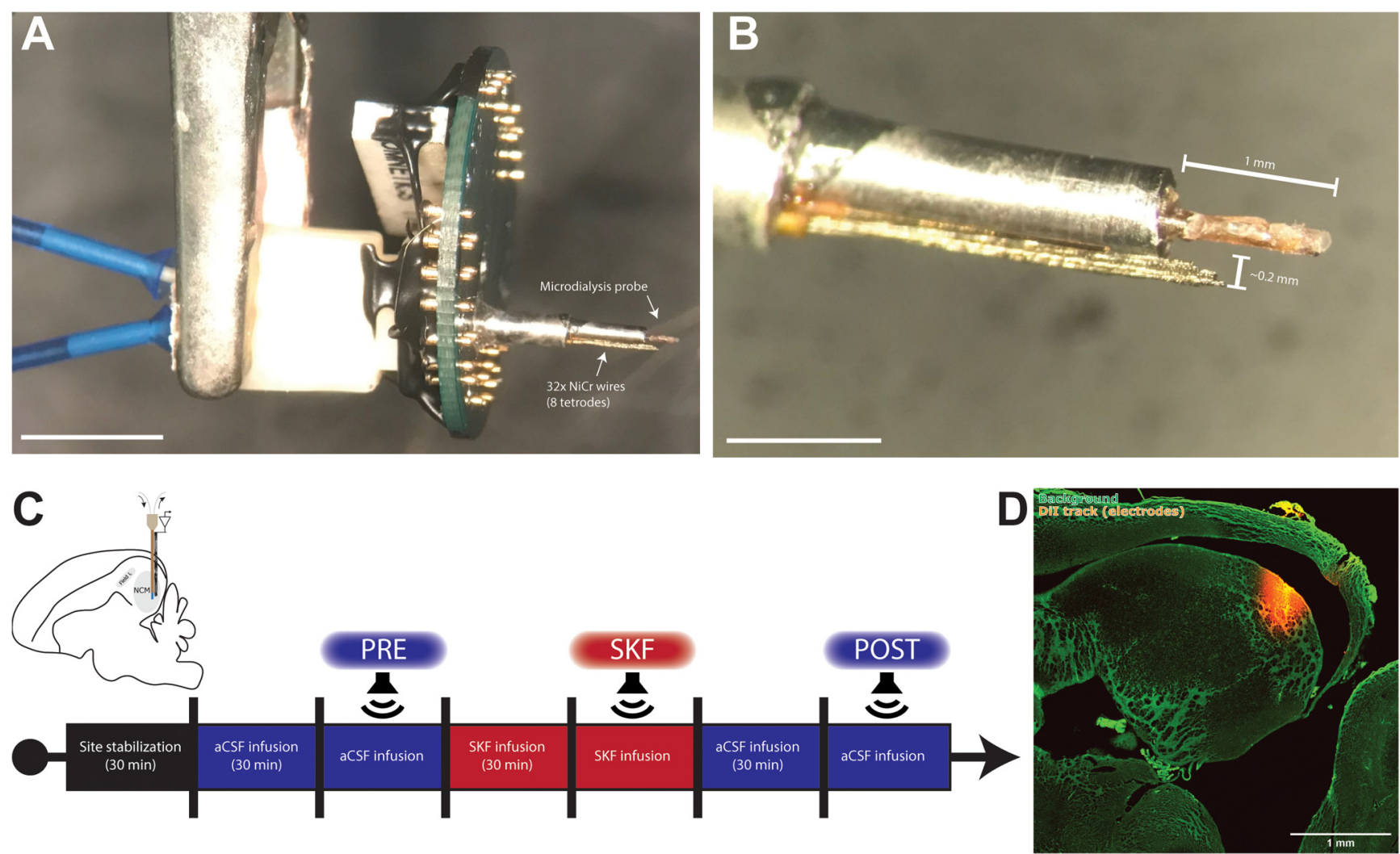

Figure 6. RetroDrive recordings, i.e., acute multichannel electrophysiology microdrive coupled to a microdialysis probe for drug delivery through retrodialysis. $A$, RetroDrives were constructed using a custom electrode interface board, a 32-channel Omnetics connector, a CMA11 (Harvard Apparatus) microdialysis probe, and 32 $\times 12.5$ - $\mu$ m-diameter NiCr wires spun into tetrodes. Scale bar: $5 \mathrm{~mm}$. B, Tetrodes were $\sim 0.2 \mathrm{~mm}$ distant from the probe and cut to extend to approximately half the length of the probe. Scale bar: $1 \mathrm{~mm}$. $\boldsymbol{C}$, Timeline for drug infusions and playbacks starting after auditory site localization within NCM. D, Electrode track confirmation. Before insertion in the brain, electrodes were dipped in Dil-594 (6.25\% in 100\% ethanol) for posthumous site confirmation.

term memory formation (Chew et al., 1996; Lu and Vicario, 2017). Therefore, we asked whether D1R activation would result in changes in adaptation to novel stimuli. Trial-by-trial stimulus firing rates were used for deriving adaptation slopes (see Materials and Methods). Eight cells (three BS1, five BS2) had to be removed from the analyses, because firing rate on the first trial used for the regression (trial 6; see Materials and Methods) was 0 during either PRE or SKF playbacks. Representative rasters and corresponding slopes of an NS1 cell is shown in Figure $8 A$. Figure $8 B$ depicts the slope through the normalized firing rate of trials 6-25 averaged by cell type, for all cell types. GLM/ANOVA analyses showed that SKF-38393 infusion reduced adaptation (i.e., shallower regression slopes), regardless of cell type (treatment: $\chi^{2}(3)=8.278, p=0.004$; cell type: $\chi^{2}(3)=20.069$, $p<0.001$; treatment $\times$ cell type: $\chi^{2}(3)=5.244, p=0.155$; Fig. $8 C)$. Note, however that this effect seems to be driven by all cell types but for BS2, which on average appears to remain unchanged by SKF. In fact, BS2 cells were the only group that retained non-zero slopes during SKF treatment (one-sample Wilcoxon signed-rank tests vs 0 ; NS1: $p=0.308$; NS2: $p=0.549$; BS1: $p=0.052$; BS2: $p<0.001$ ).

Importantly, the \%-change in slopes did not correlate with the $\%$-change in spontaneous firing (GLM/ANOVA; Spontaneous \%-change: $\chi^{2}(1)=1.126, p=0.289$; cell type: $\chi^{2}(3)=2.723, p=$ 0.436 ; spontaneous $\%$-change $\times$ cell type: $\left.\chi^{2}(3)=3.451, p=0.327\right)$ or stimulus firing (GLM/ANOVA; Stimulus \%-change: $\chi^{2}(1)=$ $0.800, p=0.371$; cell type: $\chi^{2}(3)=2.764, p=0.430$; stimulus $\%$-change $\times$ cell type: $\chi^{2}(3)=2.765, p=0.429$ ), suggesting that lower slopes are not predicted by firing rate "floor-effect" (data not shown).
Therefore, D1R activation flattens the SSA slopes of NCM neurons, consistent with the hypothesis that adaptation and memory formation in NCM are modulated by the local activation of D1Rs.

\section{Discussion}

In this study, we show that dopamine D1Rs are involved in the auditory association process and modulate excitatory/inhibitory balance in the secondary association pallium (NCM) of a songbird. We show that D1R protein is prevalent in NCM neurons (especially in $\mathrm{ARO}+, \mathrm{GABA}+$, and $\mathrm{PV}+$ populations). These $\mathrm{D} 1 \mathrm{R}+$ neurons specifically express the plasticity marker EGR1 after classical conditioning. Furthermore, D1R activation regulates NCM circuit excitability in vitro and alters responses and adaptation to song playback in vivo. Together, these pieces of evidence are consistent with the hypothesis that dopamine is an important modulator of complex sensory function and a possible substrate for reinforcement learning.

Dopamine signaling modulates auditory association learning in mammalian primary auditory cortex (Bao et al., 2001; Schicknick et al., 2008, 2012; Reichenbach et al., 2015). In these studies, dopamine activation was paired with simple stimuli such as pure and frequency-modulated tones. However, there is limited work on the role of dopamine in modulating complex auditory signal processing, in high-order cortical/pallial structures. In humans, systemic dopamine-enhancing treatments enhanced auditory language learning (Breitenstein et al., 2004; Knecht et al., 2004). Here, we show that dopamine signaling in high-order 
A
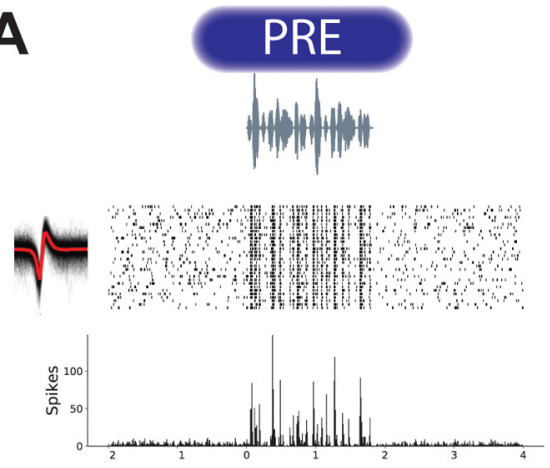

th
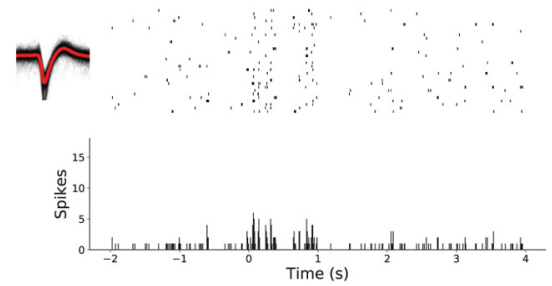

B
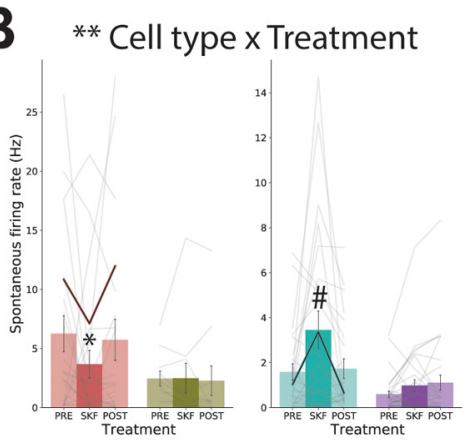

E
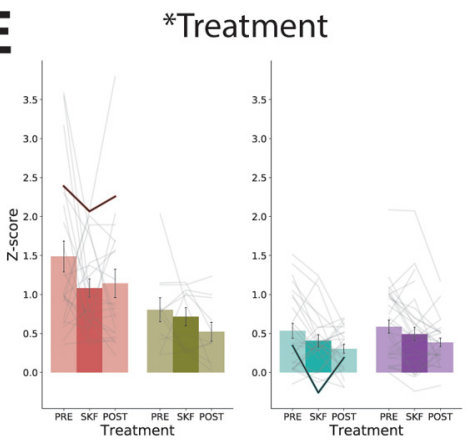

C

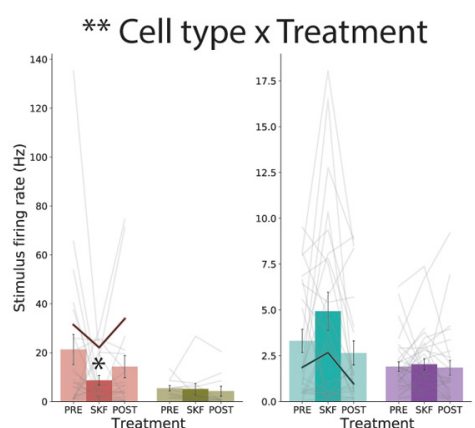

SKF
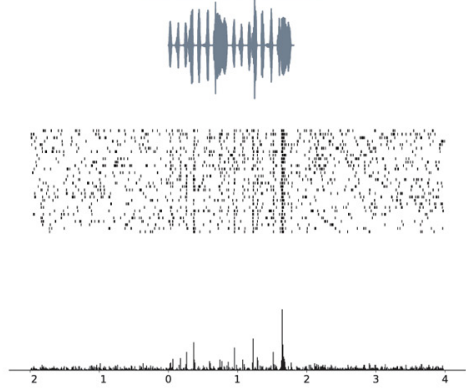

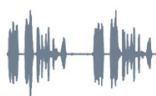
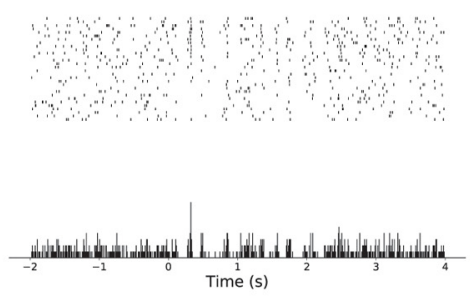

D

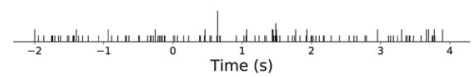

POST
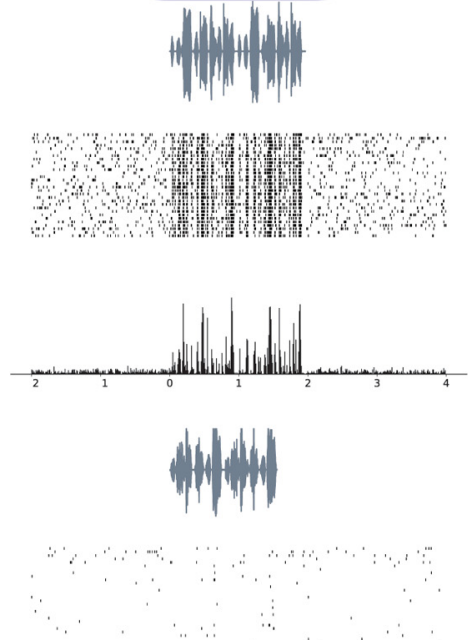

F

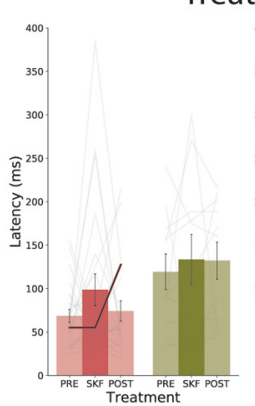

*Treatment

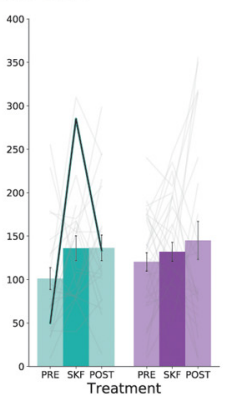

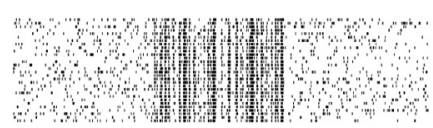

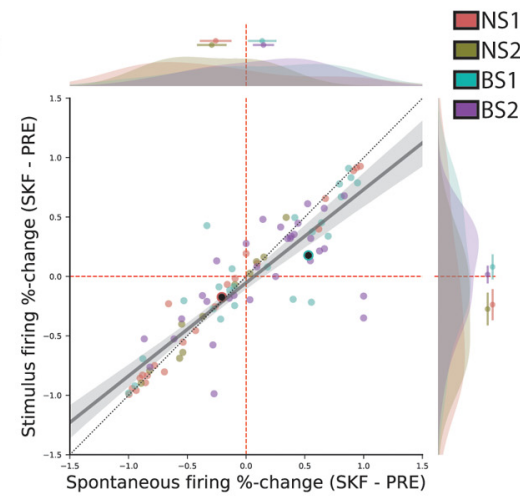

G

G **Treatment

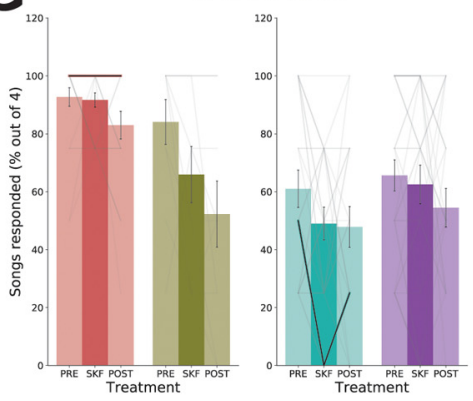

Figure 7. Effects of D1R activation in vivo on firing characteristics of NCM neurons. $A$, Representative PSTHs of an NS1 (top) and a BS1 (bottom) cell in response to SKF. Note the change in stimulus and spontaneous firing in NS1 and BS1, respectively, because of SKF-38393 (0.2 mM). Representative cells are highlighted in all plots according to their cell type. $\boldsymbol{B}$, Spontaneous firing rate. D1R agonist reduced firing of NS1 but increased firing of BS1 cells (trend in full GLM model, but significant in restricted model). C, Stimulus firing rate. D1R agonist reduced firing of NS1 cells. $\boldsymbol{D}$, Correlation between spontaneous and stimulus \%-change during SKF infusion accompanied by kernel-density estimations of the distributions along single-axis with mean \pm SEM on top. Regression line across all data points is shown (solid line). Identity line is shown as a dotted line. The regression slope $95 \%$ confidence interval (shaded area) is less than and does not encompass 1, indicating that spontaneous firing changes are higher than stimulus firing changes. $\boldsymbol{E}$, D1R agonist reduced $z$ score, $(\boldsymbol{F})$ increased latency and $(\boldsymbol{G})$ decreased responsiveness of NCM cells. Relevant statistical effects are highlighted on top of each plot. Post hoc Tukey's test results are displayed on the plots when interaction was significant; $\# p<0.1,{ }^{*} p<0.05$, ${ }^{* *} p<0.01$.

sensory pallium can modulate complex auditory signal encoding by shifting inhibitory-excitatory balance.

A distinct feature of NCM among the songbird auditory forebrain nuclei is the dense labeling for somatic ARO, an enzyme that mediates conversion of testosterone into estradiol (E2;
Saldanha et al., 2000). Many studies describe the important roles of NCM E2 in social interactions and modulation of neural responses (Maney et al., 2006; Remage-Healey et al., 2008, 2010; Remage-Healey and Joshi, 2012; Lampen et al., 2017; Krentzel et al., 2020). We recently showed that blocking ARO locally in 
A
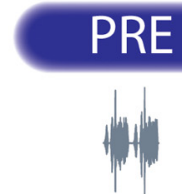

标

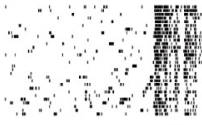

${ }_{300} \quad \stackrel{\downarrow}{\downarrow}$ Slope $=-3.81$
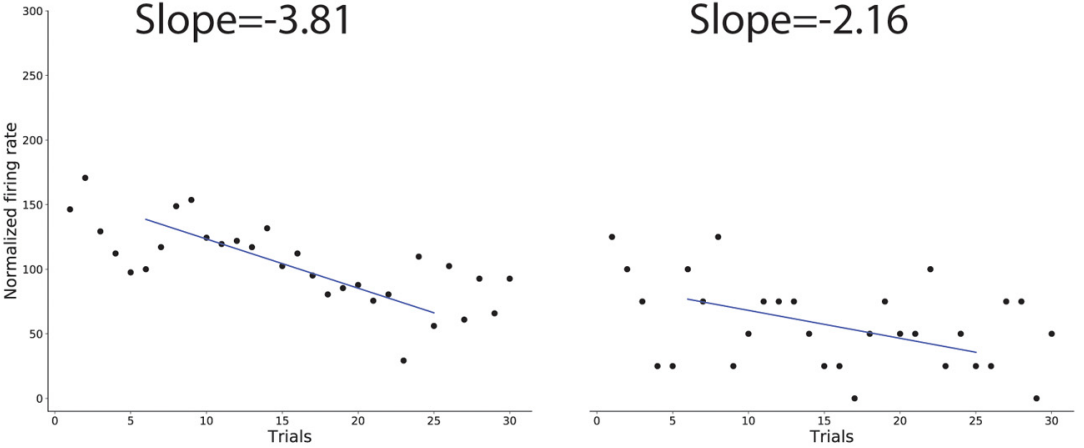

B

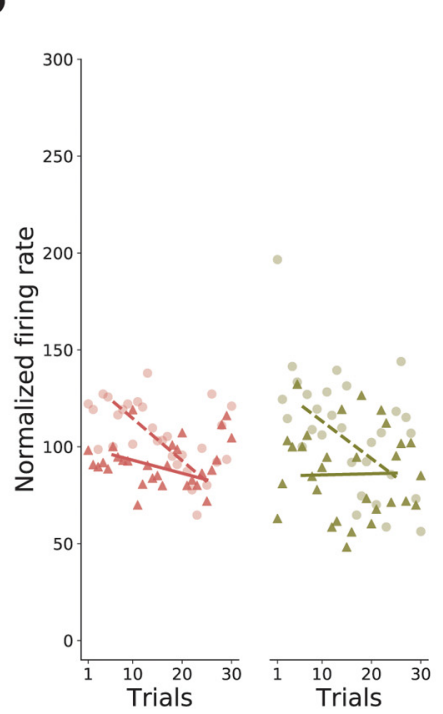

SKF
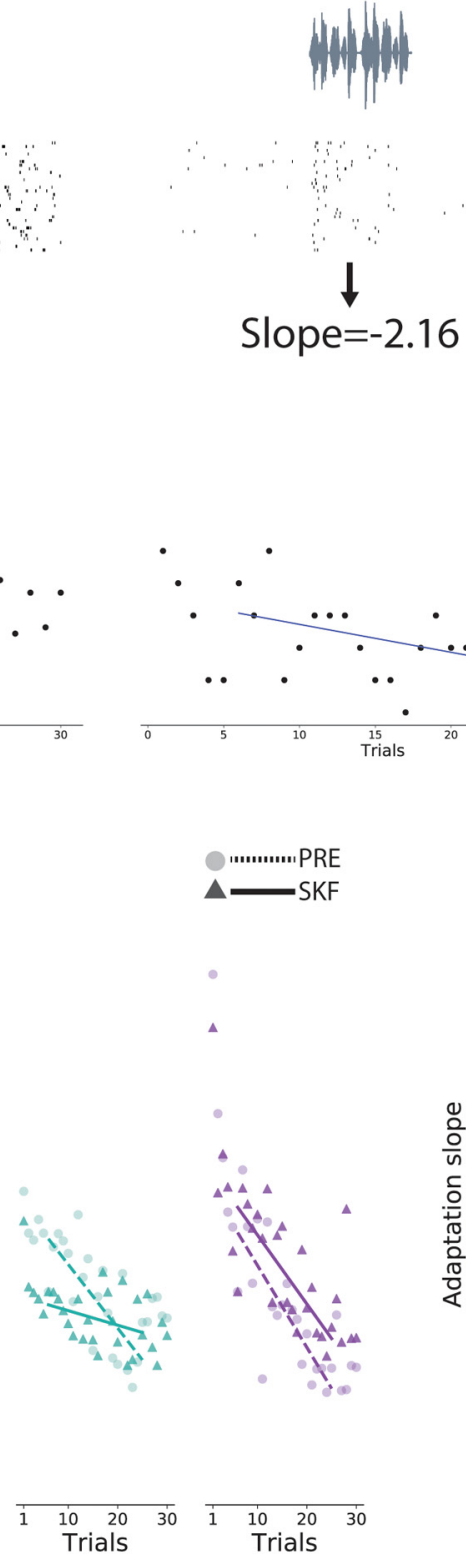

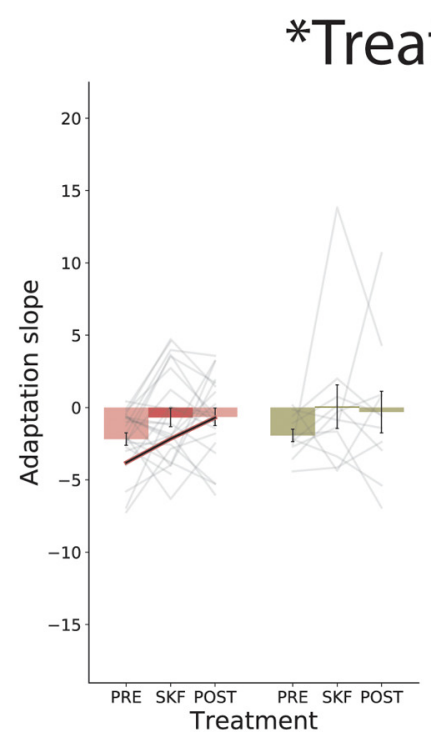

POST
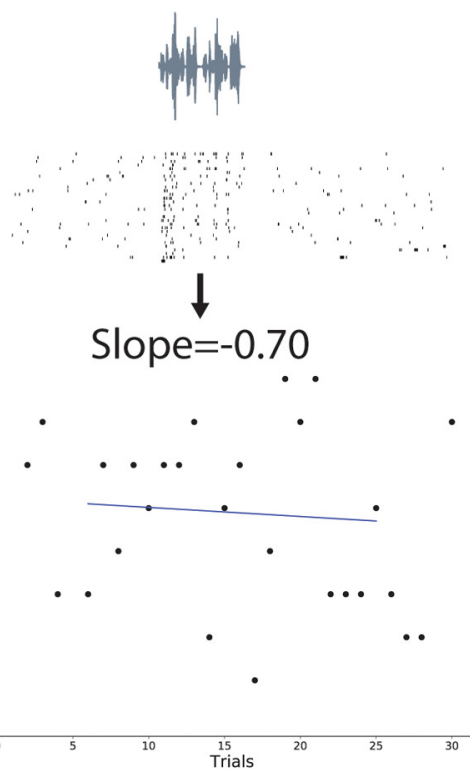

$10 \quad 15$

*Treatment

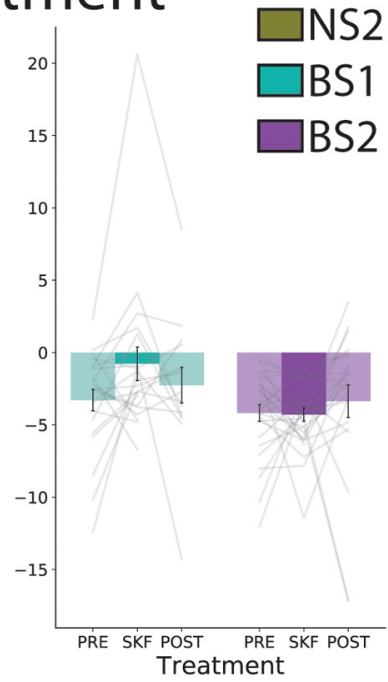

Figure 8. D1R activation disrupts neuronal SSA in vivo. $\boldsymbol{A}$, Representative PSTHs from a single NS1 highlighting a decrease in adaptation slope to a new stimulus because of SKF-38393 (0.2 $\mathrm{mm}$ ) treatment. $\boldsymbol{B}$, The slope through the normalized firing rate of trials $6-25$ averaged by cell type. $\boldsymbol{C}$, Adaptation slopes quantifications show a reduction in the slope because of SKF-38393. Relevant statistical effect is displayed on the top of the plot; ${ }^{*} p<0.05$.

NCM slows auditory association learning in an operant task (Macedo-Lima and Remage-Healey, 2020), which led to the hypothesis that dopamine interacts with E2 signaling in NCM to support association learning. In the present study, we provide anatomic evidence to support this hypothesis (Fig. 1C), suggesting that E2 and dopamine signaling act in tandem to modulate learning and memory in the songbird auditory pallium. We hypothesize that this co-modulatory signaling could apply to other brain circuits that domiciliate both ARO and dopamine receptors, such as human auditory cortex (Yague et al., 2006).

GABAergic inhibition fundamentally controls neuronal responses to sounds (Wang et al., 2002; Razak and Fuzessery, 2009) and is required for associative learning in mammal auditory cortex (Letzkus et al., 2011). Dopamine receptors are prevalent in mammalian cortical inhibitory interneurons, especially in
$\mathrm{PV}+$ neurons (Le Moine and Gaspar, 1998). PV + cortical interneurons are generally characterized by their fast action potentials and high sustained firing frequency and play a central role in regulating microcircuit activity states and learning processes in hippocampus and cortex (Donato et al., 2013; Cardin, 2018). Here, we show that $55 \%$ of the PV-expressing neurons in NCM also express D1R. We hypothesize that many of the physiological effects we observed can be attributed to this subpopulation.

D1Rs are generally assumed to increase circuit excitability through $\mathrm{G} \alpha_{\mathrm{s}}$-protein coupling (Beaulieu and Gainetdinov, 2011). In our in vitro experiments, D1R agonist SKF-38393 caused a seemingly counterintuitive reduction in the amplitude of GABAergic and glutamatergic currents. However, D1R-mediated depression is well documented in the mammalian nucleus accumbens for both GABAergic and glutamatergic (especially 


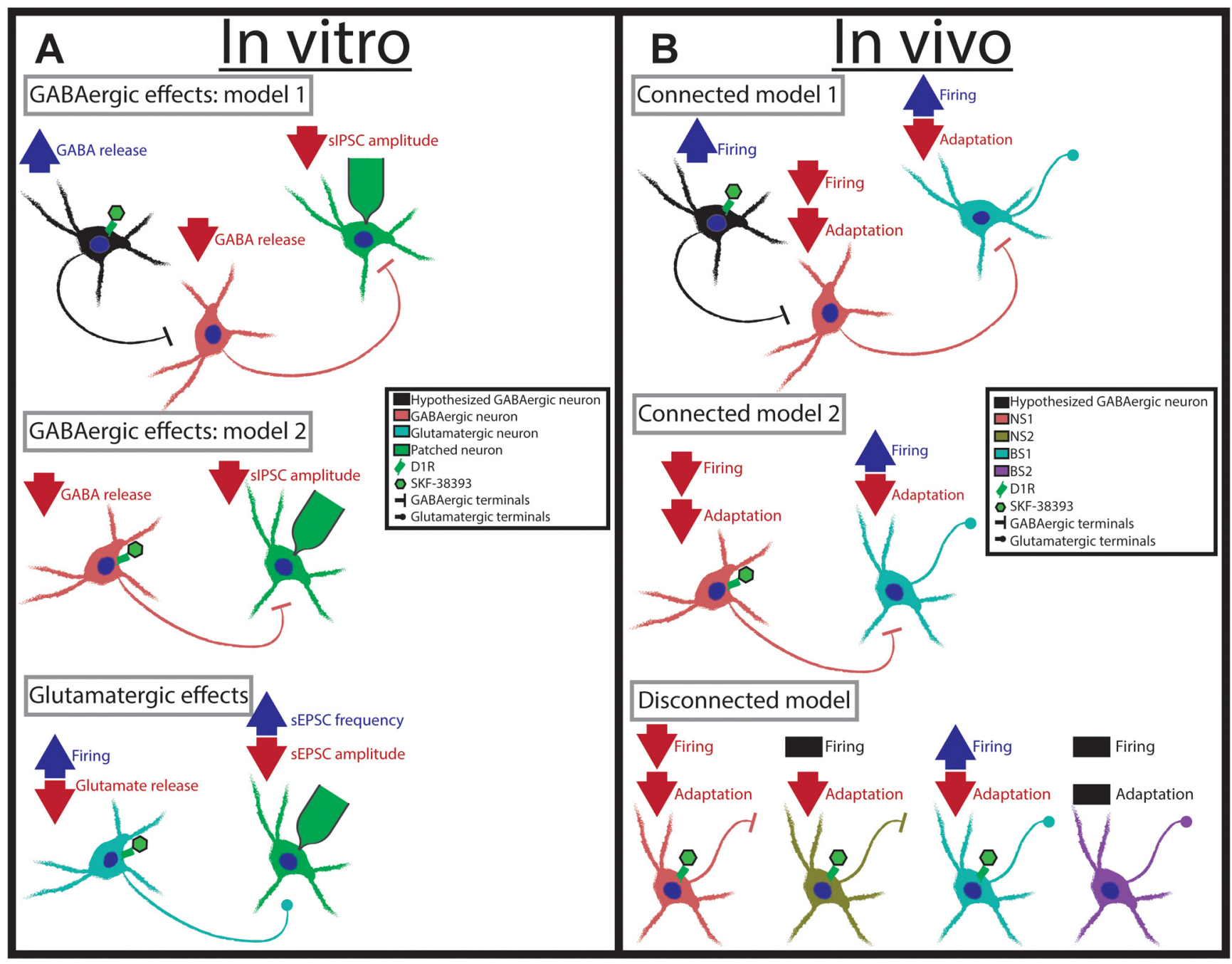

Figure 9. Network connectivity models hypothesized from current findings. SKF-38393 effects are shown by the large arrows. A, Models for in vitro results with sIPSCS (GABAergic) and SEPSCS (glutamatergic). GABAergic model 1 hypothesizes that D1R agonist binds preferentially to a GABAergic neuron upstream from the ones providing input to the recorded neuron. Binding of D1R agonist increases its GABA tone resulting in a reduction of GABA release immediately upstream of the recorded neuron. GABAergic model 2 suggests D1R effects directly cause a reduction in GABA release by the upstream neuron. Glutamatergic effects can be explained by a direct effect of D1R agonist on a glutamatergic neuron directly upstream from the recorded neuron, causing a reduction (perhaps a depletion) of glutamate release but an increase in firing rate. $\boldsymbol{B}$, Models for in vivo results. Connected models only depict cell types with changes in firing rate. Model one hypothesizes that D1R agonist binds to and excites a GABAergic neuron upstream of NS1 cells, which results in a reduction of NS1 firing and consequent disinhibition of BS1 cells. Model 2 offers a direct effect of D1R agonist on NS1 cells, resulting in a reduction in their firing, which results in disinhibition of BS1 cells. In the disconnected model, the effects observed here are because of D1R's binding to all cell types individually (except BS2, in which no effects were observed).

NMDA-mediated) synapses and is attributed to presynaptic plasticity (Pennartz et al., 1992; Zhang et al., 2014). In our in vitro proposed model (Fig. 9A), we suggest two scenarios for the reduction of GABA release: (1) D1Rs are predominantly mediating an increase in GABAergic tone by neurons upstream to those providing input to the recorded neuron, therefore reducing GABA release downstream; or (2) D1Rs are acting directly on neurons providing input to the recorded neuron causing a direct reduction in GABA release. For the reduction of glutamatergic current amplitude, we suggest a presynaptic mechanism, perhaps explained by a depletion in presynaptic glutamate stores, resulting from the increased firing (Staley et al., 1998), regulation of glutamate production (Sherman and Mott, 1985), or conversion of glutamate into GABA by glutamine decarboxylase (Neary et al., 1972) by D1Rs. Alternatively, SKF-38393 could be acting directly on the recorded neuron (postsynaptically) in NCM to result in amplitude reductions. Additional experiments with miniature events or with synaptic stimulation could help clarify these questions.
In our in vivo recordings, the characteristics of the different cell types led us to formulate hypotheses based on mammalian cortical neurons. Our NS1 neurons are highly reminiscent of mammalian fast-spiking $\mathrm{PV}+$ interneurons, exhibiting short and symmetrical action potentials, high firing, short latency to respond and low stimulus selectivity (Atallah et al., 2012; Tremblay et al., 2016). Because of the narrower waveform, lower firing rate, and longer onset latencies, we suggest that NS2 resemble late-spiking interneurons, such as somatostatin + or VIP+ interneurons (Tremblay et al., 2016). Regarding our broader waveforms, BS1 has a higher spontaneous firing rate than BS2 and BS2 is not remarkably sensitive to SKF-38393. We found $\sim 33 \%$ of NCM neurons do not express either GABA or D1Rs (Fig. 3A). Therefore, it is possible that BS2 cells are part of a circuit in which D1R-signaling does not participate to produce strong effects on the variables analyzed in this study.

We propose three models (Fig. 9B) for the effects we observed in vivo. In our "connected model 1," we suggest that the D1R 
activation might be increasing the tonic firing of GABAergic neurons upstream to NS1 cells, thus inhibiting them and disinhibiting BS1 cells. This model resembles a disinhibitory circuitry discovered in mammalian cortex for auditory associative learning, in which learning activates layer 1 inhibitory interneurons, which in turn inhibit layer $2 / 3 \mathrm{PV}+$ interneurons, thus disinhibiting pyramidal neurons. These layer 1 neurons are activated by cholinergic signaling (Letzkus et al., 2011), and are known to be 5HT3a+/VIP- interneurons (Tremblay et al., 2016). Alternatively, our "connected model 2" depicts a single synapse and inhibitory effects of SKF-38393 on NS1 cells. Finally, our "disconnected model" summarizes our findings in each cell type and depicts isolated effects of the D1R agonist. Future experiments involving genetic targeting of specific neuronal subtypes could clarify these circuit properties.

Acetylcholine has been shown to affect SSA in mammalian auditory cortex and inferior colliculus (Metherato and Weinberger, 1989; Ayala and Malmierca, 2015). However, to our knowledge, dopamine modulation of SSA in vertebrate auditory cortex/pallium has not been explored. In mammalian auditory cortex, D1R modulation improves signal detection and association learning (Schicknick et al., 2012; Happel et al., 2014). Likewise, in humans, systemic dopaminergic treatments improve auditory language associative learning (Breitenstein et al., 2004; Knecht et al., 2004). These studies suggest it is plausible that dopamine could be affecting SSA. In songbird NCM, SSA has been shown to parallel familiarity with sounds, such that novel sounds will produce more negative slopes (i.e., higher SSA) than familiar, previously adapted sounds (Chew et al., 1996). In fact, after successful behavioral association learning, learned sounds produce less SSA than novel sounds (Bell et al., 2015). Here, we provide evidence that D1Rs are involved in this process, such that pharmacological D1R activation disrupts SSA in NCM neurons.

We note that the origins of dopaminergic projection to NCM are still an open question in the field. There are eight major subpallial dopaminergic nuclei, which are fairly well conserved across vertebrates (Reiner et al., 1998). Preliminary reports suggest the caudal ventral tegmental area projects to NCM (Barr et al., 2019; Yanagihara et al., 2019), which, if confirmed, would be an exciting avenue for studying auditory reward prediction learning. Other reports suggest that the locus coeruleus (LC) projects to NCM to provide norepinephrinergic input (Ikeda et al., 2015; Chen et al., 2016). Norepinephrine is a precursor to dopamine, and LC neurons are known to release dopamine in addition to NE throughout the cortex (Devoto et al., 2005). Dopamine released by the LC onto dorsal hippocampus is involved in spatial learning and memory in rodents, independently of NE release (Kempadoo et al., 2016; Takeuchi et al., 2016). Furthermore, future studies should clarify through neuronal tract tracing which specific nuclei provide dopaminergic inputs to NCM and whether the effects observed in this study can be mimicked by dopamine release from such nuclei.

In conclusion, we show that D1R signaling shifts the excitatory-inhibitory balance in songbird pallium to modulate mechanisms involved in auditory learning and key components of auditory response, circuitry, and plasticity. We propose that D1Rs are important mediators of learning and memory in the avian sensory pallium and this mechanism could be a common feature among vertebrates.

\section{References}

Alvarez-Buylla A, Buskirk DR, Nottebohm F (1987) Monoclonal antibody reveals radial glia in adult avian brain. J Comp Neurol 264:159-170.

Atallah BV, Bruns W, Carandini M, Scanziani M (2012) Parvalbuminexpressing interneurons linearly transform cortical responses to visual stimuli. Neuron 73:159-170.

Atencio CA, Schreiner CE (2008) Spectrotemporal processing differences between auditory cortical fast-spiking and regular-spiking neurons. J Neurosci 28:3897-3910

Avey MT, Phillmore LS, MacDougall-Shackleton SA (2005) Immediate early gene expression following exposure to acoustic and visual components of courtship in zebra finches. Behav Brain Res 165:247-253.

Ayala YA, Malmierca MS (2015) Cholinergic modulation of stimulus-specific adaptation in the inferior colliculus. J Neurosci 35:12261-12272.

Bao S, Chan VT, Merzenich MM (2001) Cortical remodelling induced by activity of ventral tegmental dopamine neurons. Nature 412:79-83.

Barr HJ, Wall EM, Woolley SC (2019) Dopamine in the songbird auditory cortex shapes auditory preference. bioRxiv 761783 .

Beaulieu JM, Gainetdinov RR (2011) The physiology, signaling, and pharmacology of dopamine receptors. Pharmacol Rev 63:182-217.

Bell BA, Phan ML, Vicario DS (2015) Neural responses in songbird forebrain reflect learning rates, acquired salience, and stimulus novelty after auditory discrimination training. J Neurophysiol 113:1480-1492.

Bliss CI, Greenwood ML, White ES (1956) A rankit analysis of paired comparisons for measuring the effect of sprays on flavor. Biometrics 12:381.

Blumstein DT, Daniel JC (2007) Quantifying behavior the JWatcher way. Sunderland: Sinauer Associates Incorporated.

Boersma P, van Heuven V (2001) Speak and unSpeak with Praat. Glot Int 5:341-347.

Bolhuis JJ, Okanoya K, Scharff C (2010) Twitter evolution: converging mechanisms in birdsong and human speech. Nat Rev Neurosci 11:747-759.

Bottjer SW, Ronald AA, Kaye T (2019) Response properties of single neurons in higher level auditory cortex of adult songbirds. J Neurophysiol 121:218-237.

Breitenstein C, Wailke S, Bushuven S, Kamping S, Zwitserlood P, Ringelstein EB, Knecht S (2004) D-amphetamine boosts language learning independent of its cardiovascular and motor arousing effects. Neuropsychopharmacology 29:1704-1714.

Cardin JA (2018) Inhibitory interneurons regulate temporal precision and correlations in cortical circuits. Trends Neurosci 41:689-700.

Cazala A, Giret N, Edeline JM, Del Negro C (2019) Neuronal encoding in a high level auditory area: from sequential order of elements to grammatical structure. J Neurosci 39:6150-6161.

Charrad M, Ghazzali N, Boiteau V, Niknafs A (2014) Nbclust: an R package for determining the relevant number of clusters in a data set. J Stat Softw 61:1-36.

Chen Y, Matheson LE, Sakata JT (2016) Mechanisms underlying the social enhancement of vocal learning in songbirds. Proc Natl Acad Sci USA 113:6641-6646.

Chew SJ, Vicario DS, Nottebohm FN (1996) A large-capacity memory system that recognizes the calls and songs of individual birds. Proc Natl Acad Sci USA 93:1950-1955.

Clements JD, Bekkers JM (1997) Detection of spontaneous synaptic events with an optimally scaled template. Biophys J 73:220-229.

Devoto P, Flore G, Saba P, Fà M, Gessa GL (2005) Co-release of noradrenaline and dopamine in the cerebral cortex elicited by single train and repeated train stimulation of the locus coeruleus. BMC Neurosci 6:31.

Ding L, Perkel DJ (2002) Dopamine modulates excitability of spiny neurons in the avian basal ganglia. J Neurosci 22:5210-5218.

Donato F, Rompani SB, Caroni P (2013) Parvalbumin-expressing basket-cell network plasticity induced by experience regulates adult learning. Nature 504:272-276.

Forlano PM, Schlinger BA, Bass AH (2006) Brain aromatase: new lessons from non-mammalian model systems. Front Neuroendocrinol 27:247274.

Gadagkar V, Puzerey PA, Chen R, Baird-Daniel E, Farhang AR, Goldberg JH (2016) Dopamine neurons encode performance error in singing birds. Science 354:1278-1354.

Galoch Z, Bischof HJ (2007) Behavioural responses to video playbacks by zebra finch males. Behav Processes 74:21-26.

Happel MFK, Deliano M, Handschuh J, Ohl FW (2014) Dopamine-modulated recurrent corticoefferent feedback in primary sensory cortex 
promotes detection of behaviorally relevant stimuli. J Neurosci 34:12341247.

Hromádka T, DeWeese MR, Zador AM (2008) Sparse representation of sounds in the unanesthetized auditory cortex. PLoS Biol 6:e16.

Ikeda MZ, Jeon SD, Cowell RA, Remage-Healey L (2015) Norepinephrine modulates coding of complex vocalizations in the songbird auditory cortex independent of local neuroestrogen synthesis. J Neurosci 35:93569368.

Jarvis ED (2019) Evolution of vocal learning and spoken language. Science 366:50-54.

Kempadoo KA, Mosharov EV, Choi SJ, Sulzer D, Kandel ER (2016) Dopamine release from the locus coeruleus to the dorsal hippocampus promotes spatial learning and memory. Proc Natl Acad Sci USA 113:14835-14840

Knecht S, Breitenstein C, Bushuven S, Wailke S, Kamping S, Flöel A, Zwitserlood P, Ringelstein EB (2004) Levodopa: faster and better word learning in normal humans. Ann Neurol 56:20-26.

Krentzel AA, Ikeda MZ, Oliver TJ, Koroveshi E, Remage-Healey L (2020) Acute neuroestrogen blockade attenuates song-induced immediate early gene expression in auditory regions of male and female zebra finches. J Comp Physiol A Neuroethol Sens Neural Behav Physiol 206:15-31.

Kubikova L, Wada K, Jarvis ED (2010) Dopamine receptors in a songbird brain. J Comp Neurol 518:741-769.

Lampen J, McAuley JD, Chang SE, Wade J (2017) ZENK induction in the zebra finch brain by song: relationship to hemisphere, rhythm, oestradiol and sex. J Neuroendocrinol 29:10.

Le Moine C, Gaspar P (1998) Subpopulations of cortical GABAergic interneurons differ by their expression of D1 and D2 dopamine receptor subtypes. Brain Res Mol Brain Res 58:231-236.

Leblois A, Wendel BJ, Perkel DJ (2010) Striatal dopamine modulates basal ganglia output and regulates social context-dependent behavioral variability through D1 receptors. J Neurosci 30:5730-5743.

Lee V, Pawlisch BA, Macedo-Lima M, Remage-Healey L (2018) Norepinephrine enhances song responsiveness and encoding in the auditory forebrain of male zebra finches. J Neurophysiol 119:209-220.

Letzkus JJ, Wolff SBE, Meyer EMM, Tovote P, Courtin J, Herry C, Lüthi A (2011) A disinhibitory microcircuit for associative fear learning in the auditory cortex. Nature 480:331-335.

Lu K, Vicario DS (2017) Familiar but unexpected: effects of sound context statistics on auditory responses in the songbird forebrain. J Neurosci 37:12006-12017.

Lüscher C, Malenka RC (2012) NMDA receptor-dependent long-term potentiation and long-term depression (LTP/LTD). Cold Spring Harb Perspect Biol 4:a005710-16.

Macedo-Lima M, Remage-Healey L (2020) Auditory learning in an operant task with social reinforcement is dependent on neuroestrogen synthesis in the male songbird auditory cortex. Horm Behav 121:104713.

Maney DL, Cho E, Goode CT (2006) Estrogen-dependent selectivity of genomic responses to birdsong. Eur J Neurosci 23:1523-1529.

Matheson LE, Sakata JT (2015) Catecholaminergic contributions to vocal communication signals. Eur J Neurosci 41:1180-1194.

Matragrano LL, Beaulieu M, Phillip JO, Rae AI, Sanford SE, Sockman KW, Maney DL (2012) Rapid effects of hearing song on catecholaminergic activity in the songbird auditory pathway. PLoS One 7:e39388.

Mello CV, Ribeiro S (1998) ZENK protein regulation by song in the brain of songbirds. J Comp Neurol 393:426-438.

Mello CV, Clayton DF (1994) Song-induced ZENK gene expression in auditory pathways of songbird brain and its relation to the song control system. J Neurosci 14:6652-6666.

Mello CV, Vicario DS, Clayton DF (1992) Song presentation induces gene expression in the songbird forebrain. Proc Natl Acad Sci USA 89:68186822.

Metherato R, Weinberger NM (1989) Acetylcholine produces stimulus-specific receptive field alterations in cat auditory cortex. Brain Res 480:372377.

Müller CM, Leppelsack H-J (1985) Feature extraction and tonotopic organization in the avian auditory forebrain. Exp Brain Res 59:587-599.

Neary JT, Meneely RL, Grever MR, Diven WF (1972) The interactions between biogenic amines and pyridoxal, pyridoxal phosphate, and pyridoxal kinase. Arch Biochem Biophys 151:42-47.
Nguyen T, Bhatti A, Khosravi A, Haggag S, Creighton D, Nahavandi S (2015) Automatic spike sorting by unsupervised clustering with diffusion maps and silhouettes. Neurocomputing 153:199-210.

Ono S, Okanoya K, Seki Y (2016) Hierarchical emergence of sequence sensitivity in the songbird auditory forebrain. J Comp Physiol A Neuroethol Sens Neural Behav Physiol 202:163-183.

Pachitariu M, Steinmetz N, Kadir S, Carandini M, Harris KD (2016) Kilosort: realtime spike-sorting for extracellular electrophysiology with hundreds of channels. bioRxiv 061481:1-14.

Pennartz CMA, Dolleman-Van der Weel MJ, Kitai ST, Lopes Da Silva FH (1992) Presynaptic dopamine D1 receptors attenuate excitatory and inhibitory limbic inputs to the shell region of the rat nucleus accumbens studied in vitro. J Neurophysiol 67:1325-1334.

Phan ML, Pytte CL, Vicario DS (2006) Early auditory experience generates long-lasting memories that may subserve vocal learning in songbirds. Proc Natl Acad Sci USA 103:1088-1093.

Razak KA, Fuzessery ZM (2009) GABA shapes selectivity for the rate and direction of frequency-modulated sweeps in the auditory cortex. J Neurophysiol 102:1366-1378.

Reichenbach N, Herrmann U, Kähne T, Schicknick H, Pielot R, Naumann M, Dieterich DC, Gundelfinger ED, Smalla KH, Tischmeyer W (2015) Differential effects of dopamine signalling on long-term memory formation and consolidation in rodent brain. Proteome Sci 13:13.

Reiner A, Karle EJ, Anderson KD, Medina L (1994) Catecholaminergic perikarya and fibers in the avian nervous system. In: Phylogeny and development of catecholamine systems in the CNS of vertebrates, pp 135-181. Cambridge: Cambridge University Press.

Reiner A, Medina L, Veenman CL (1998) Structural and functional evolution of the basal ganglia in vertebrates. Brain Res Brain Res Rev 28:235-285.

Remage-Healey L, Joshi NR (2012) Changing neuroestrogens within the auditory forebrain rapidly transform stimulus selectivity in a downstream sensorimotor nucleus. J Neurosci 32:8231-8241.

Remage-Healey L, Maidment NT, Schlinger BA (2008) Forebrain steroid levels fluctuate rapidly during social interactions. Nat Neurosci 11:13271334.

Remage-Healey L, Coleman MJ, Oyama RK, Schlinger BA (2010) Brain estrogens rapidly strengthen auditory encoding and guide song preference in a songbird. Proc Natl Acad Sci USA 107:3852-3857.

Rothman JS, Silver RA (2018) Neuromatic: an integrated open-source software toolkit for acquisition, analysis and simulation of electrophysiological data. Front Neuroinform 12:1-21.

Saldanha CJ, Tuerk MJ, Kim YH, Fernandes AO, Arnold AP, Schlinger BA (2000) Distribution and regulation of telencephalic aromatase expression in the zebra finch revealed with a specific antibody. J Comp Neurol 423:619-630.

Saldanha CJ, Schlinger BA, Micevych PE, Horvath TL (2004) Presynaptic Nmethyl-D-aspartate receptor expression is increased by estrogen in an aromatase-rich area of the songbird hippocampus. J Comp Neurol 469:522-534.

Sanford SE, Lange HS, Maney DL (2010) Topography of estradiol-modulated genomic responses in the songbird auditory forebrain. Dev Neurobiol 70:73-86.

Schicknick H, Schott BH, Budinger E, Smalla KH, Riedel A, Seidenbecher CI, Scheich H, Gundelfinger ED, Tischmeyer W (2008) Dopaminergic modulation of auditory cortex-dependent memory consolidation through mTOR. Cereb Cortex 18:2646-2658.

Schicknick H, Reichenbach N, Smalla KH, Scheich H, Gundelfinger ED, Tischmeyer W (2012) Dopamine modulates memory consolidation of discrimination learning in the auditory cortex. Eur J Neurosci 35:763774.

Schmidt MF, Ding L (2014) Achieving perfection through variability: the basal ganglia helped me do it! Neuron 82:6-8.

Schneider DM, Woolley SMN (2013) Sparse and background-invariant coding of vocalizations in auditory scenes. Neuron 79:141-152.

Sherman AD, Mott J (1985) Amphetamine stimulation of glutaminase is blocked by neuroleptics. Life Sci 36:1163-1167.

Staley KJ, Longacher M, Bains JS, Yee A (1998) Presynaptic modulation of CA3 network activity. Nat Neurosci 1:201-209.

Takeuchi T, Duszkiewicz AJ, Sonneborn A, Spooner PA, Yamasaki M, Morris RGM, Watanabe M, Smith CC, Fernández G, Deisseroth K, Robert W (2016) Locus coeruleus and dopaminergic consolidation of everyday memory. Nature 537:357-362. 
Tanaka M, Sun F, Li Y, Mooney R (2018) A mesocortical dopamine circuit enables the cultural transmission of vocal behaviour. Nature 563:117120

Tibshirani R, Walther G, Hastie T (2001) Estimating the number of clusters in a data set via the gap statistic. J R Stat Soc Series B Stat Methodol 63:411-423.

Tremblay R, Lee S, Rudy B (2016) GABAergic interneurons in the neocortex: from cellular properties to circuits. Neuron 91:260-292.

Tsunada J, Lee JH, Cohen YE (2012) Differential representation of auditory categories between cell classes in primate auditory cortex. J Physiol 590:3129-3139.

Vahaba DM, Macedo-Lima M, Remage-Healey L (2017) Sensory coding and sensitivity to local estrogens shift during critical period milestones in the auditory cortex of male songbirds. eNeuro 4:ENEURO.0317-17.2017.

von Eugen K, Tabrik S, Güntürkün O, Ströckens F (2020) A comparative analysis of the dopaminergic innervation of the executive caudal nidopallium in pigeon, chicken, zebra finch, and carrion crow. J Comp Neurol 528:2929-2955.
Wang J, McFadden SL, Caspary D, Salvi R (2002) Gamma-aminobutyric acid circuits shape response properties of auditory cortex neurons. Brain Res 944:219-231.

Wu GK, Arbuckle R, Liu B. h, Tao HW, Zhang LI (2008) Lateral sharpening of cortical frequency tuning by approximately balanced inhibition. Neuron 58:132-143.

Yague JG, Muñoz A, De Monasterio-Schrader P, DeFelipe J, Garcia-Segura LM, Azcoitia I (2006) Aromatase expression in the human temporal cortex. Neuroscience 138:389-401.

Yanagihara S, Yazaki-Sugiyama Y (2016) Auditory experience-dependent cortical circuit shaping for memory formation in bird song learning. Nat Commun 7:11946.

Yanagihara S, Ikebuchi M, Mori C, Tachibana RO, Okanoya K (2019) Social context modulates auditory activity in a songbird VTA/SNc. Program No. 233.03. 2019 Neuroscience Meeting Planner. Chicago.

Zhang L, Bose P, Warren RA (2014) Dopamine preferentially inhibits NMDA receptor-mediated EPSCs by acting on presynaptic D1 receptors in nucleus accumbens during postnatal development. PLoS One 9: e86970. 